\title{
Natura e patria. \\ I congressi della Società Italiana di Scienze Naturali nel processo di costruzione dell'identità nazionale
}

\begin{abstract}
Riassunto - La Società Italiana di Scienze Naturali (SISN), fondata a Milano nel 1855 prima con il nome di "Società geologica residente in Milano" e poi, dal 22 gennaio 1860, con il nome che la contraddistingue attualmente, ebbe un ruolo attivo nel processo di costruzione dell'identità nazionale all'indomani dell'Unità d'Italia. Prima società naturalistica italiana, essa manifestò questa vocazione soprattutto attraverso i congressi annuali, organizzati tra il 1864 e il 1906 in varie città della Penisola. Le riunioni straordinarie fuori sede avevano finalità ben precise: l'esplorazione geologica e naturalistica del nuovo territorio unificato, in gran parte ancora sconosciuto; la legittimazione della figura del naturalista come scienziato professionista; la divulgazione della scienza come motore di progresso del paese; l'affermazione della SISN come centro di riferimento per tutti i naturalisti italiani. Il presente lavoro propone dunque una lettura della storia della Società dalle origini al 1906 attraverso la lente dei congressi postunitari.
\end{abstract}

Parole chiave: Società geologica residente in Milano, Società Italiana di Scienze Naturali, Museo Civico di Storia Naturale di Milano, congressi, costruzione della nazione, patria, "Risorgimento".

\begin{abstract}
Nature and homeland. The congresses of the "Società Italiana di Scienze Naturali" in the building process of Italy's national identity.

The "Società Italiana di Scienze Naturali" (SISN), founded in Milan in 1855, first as "Società Geologica residente in Milano" and since 22 January 1860 with its present name, played an active role in the building process of the national identity after the unification of Italy. Being the first naturalistic society in Italy, it manifested this vocation particularly through its annual congresses, organized between 1864 and 1906 in various cities of the Country. The special off-site meetings had well defined purposes: the geological and naturalistic exploration of the newly unified territory, still greatly undocumented; the justification of the naturalist as a professional scientist; the spread of science as the driving force of progress for Italy; the assertion of the SISN as a reference centre for all Italian naturalists.

The present paper proposes reading the history of the "Società" from its origins to 1906 through the looking glass of the post-unification congresses.
\end{abstract}

Key words: Società Geologica residente in Milano, Società Italiana di Scienze Naturali, Museo Civico di Storia Naturale di Milano, congresses, nation building, homeland, "Risorgimento".

\footnotetext{
*Assegnista di ricerca, Università degli Studi di Milano-Bicocca, Piazza dell'Ateneo Nuovo 1, 20126 Milano, Italia; e-mail: paola.zocchi@unimib.it

Questa ricerca è stata realizzata nell'ambito del progetto "Nascita di una comunità poliscientifica. Istituzioni, attori e ideali di un secolo di cultura scientifica a Milano, 1863-1963", con il contributo di Fondazione Cariplo. Il presente lavoro è una versione rivista e corretta dell'articolo pubblicato nel sito Milano città delle scienze (www.milanocittadellescienze.it).
} 


\section{Le origini}

La storia della Società Italiana di Scienze Naturali (SISN), fondata nel 1856 con il nome di Società Geologica residente in Milano (SGM), fu in origine una storia italo-austriaca. Anche se all'indomani della prima guerra d'indipendenza i rapporti tra l'Austria e il Regno Lombardo-Veneto apparivano tutt'altro che distesi, i rispettivi uomini di scienza avevano frequenti contatti epistolari e di collaborazione scientifica.

Nel 1849 due eventi pressoché concomitanti posero le premesse per la nascita della Società. Quell'anno, infatti, un ingegnere milanese, Ambrogio Robiati $(\uparrow 1861)$, titolare di un omonimo istituto d'istruzione privato nel capoluogo lombardo, inaugurò un corso libero di geologia, affidandolo al naturalista Giuseppe Balsamo Crivelli (1800-1874), già insegnante di storia naturale nei licei milanesi di Sant'Alessandro e Porta Nuova, conservatore del Museo Civico di Storia Naturale di Milano (MSNM) (fondato nel 1838) e successivamente professore di storia naturale all'Università di Pavia.

Parallelamente, nel dicembre 1849, venne fondato a Vienna l'I.R. Istituto geologico, alla cui direzione fu nominato Wilhelm Haidinger (1795-1871) ${ }^{1}$, già direttore del locale Museo montanistico. Fu proprio Haidinger, nel maggio 1850, a contattare il direttore del Museo civico milanese Giorgio Jan (1791-1866), chiedendogli ufficialmente di istituire a Milano una Società geologica che operasse di concerto con l'istituto viennese, nel quadro più ampio della politica austriaca volta alla localizzazione e allo sfruttamento delle risorse geologiche dell'impero.

In quel momento, tuttavia, la direzione del Museo civico di Milano aveva una questione in sospeso con l'Austria: al di là della situazione politica, irrimediabilmente compromessa dopo il Quarantotto, il Museo stava infatti cercando di ottenere la proprietà dell'importante raccolta di minerali e fossili cosiddetta di Santa Teresa $^{2}$, che dal 1848 custodiva soltanto in deposito. Decise quindi di subordinare la fondazione della Società geologica alla definitiva cessione della raccolta da parte del governo austriaco, sospendendo di fatto ogni trattativa ${ }^{3}$, poiché il passaggio di proprietà fu effettuato solo molti anni dopo, nel 1857.

Nel gennaio 1855, tuttavia, Haidinger ricevette il programma dei corsi dell'Istituto Robiati, venendo così a conoscenza del corso di geologia da lui istituito nel 1849. Ritenne allora doveroso inviargli in dono una serie di volumi per la biblioteca e in tale occasione Robiati - agendo in modo del tutto indipendente dal MSNM - inserì nella sua lettera di ringraziamento la proposta di istituire a Milano una Società geologica, ottenendo di fatto l'appoggio dell'Istituto di Vienna ${ }^{4}$.

Le complesse vicende che tra il 1855 e il 1856 portarono alla fondazione della $\mathrm{SISN}^{5}$ rivelano quindi, almeno inizialmente, la scarsa volontà dei naturalisti del Museo civico milanese di collaborare con il governo austriaco. Una volta fondata la società, tuttavia, essi decisero di subentrare a Robiati - che non aveva le competenze necessarie - alla guida della nuova associazione, la cui sede - prima ubicata presso il suo istituto e dal 1858 presso l'Osservatorio astronomico di Brera - fu ospitata dal 1866 all'interno del Museo stesso.

Il forte legame con il Museo fu del resto evidente fin dall'inizio, tanto che già nel Regolamento generale del 3 novembre 1857 si specificava che in caso di scioglimento della Società, "le collezioni e gli oggetti appartenenti alla medesima verranno donati alla città di Milano, pel Museo civico, dove saranno conservati in apposita sede per istruzione della gioventù, sotto il titolo di Collezioni della Società Geologica di Milano"6. 
Inoltre, fin dal 1856, lo zoologo Emilio Cornalia (1824-1882) (Fig. 1), all'epoca assistente alla direzione del Museo e futuro direttore dello stesso fino alla morte, figurava nella Commissione presidenziale della SISN in qualità di segretario e poi di presidente, carica quest'ultima che gli fu confermata nel 1858 e che mantenne per tutta la vita.

Nonostante il suo forte radicamento nella realtà locale milanese e lo stretto legame con il Comune, a cui faceva capo il Museo, la SISN ebbe fin dalle origini un ruolo fondamentale nell'aggregazione dei naturalisti della penisola, in quanto prima società geologica fondata in Italia. La sua attività, dopo lunghi ritardi dovuti all'inesperienza di Robiati, alla lentezza della burocrazia austriaca e alla diffidenza delle autorità nei confronti dell'associazionismo in generale, fu inaugurata ufficialmente nel gennaio 1859, pochi mesi prima dell'annessione della Lombardia al Piemonte.

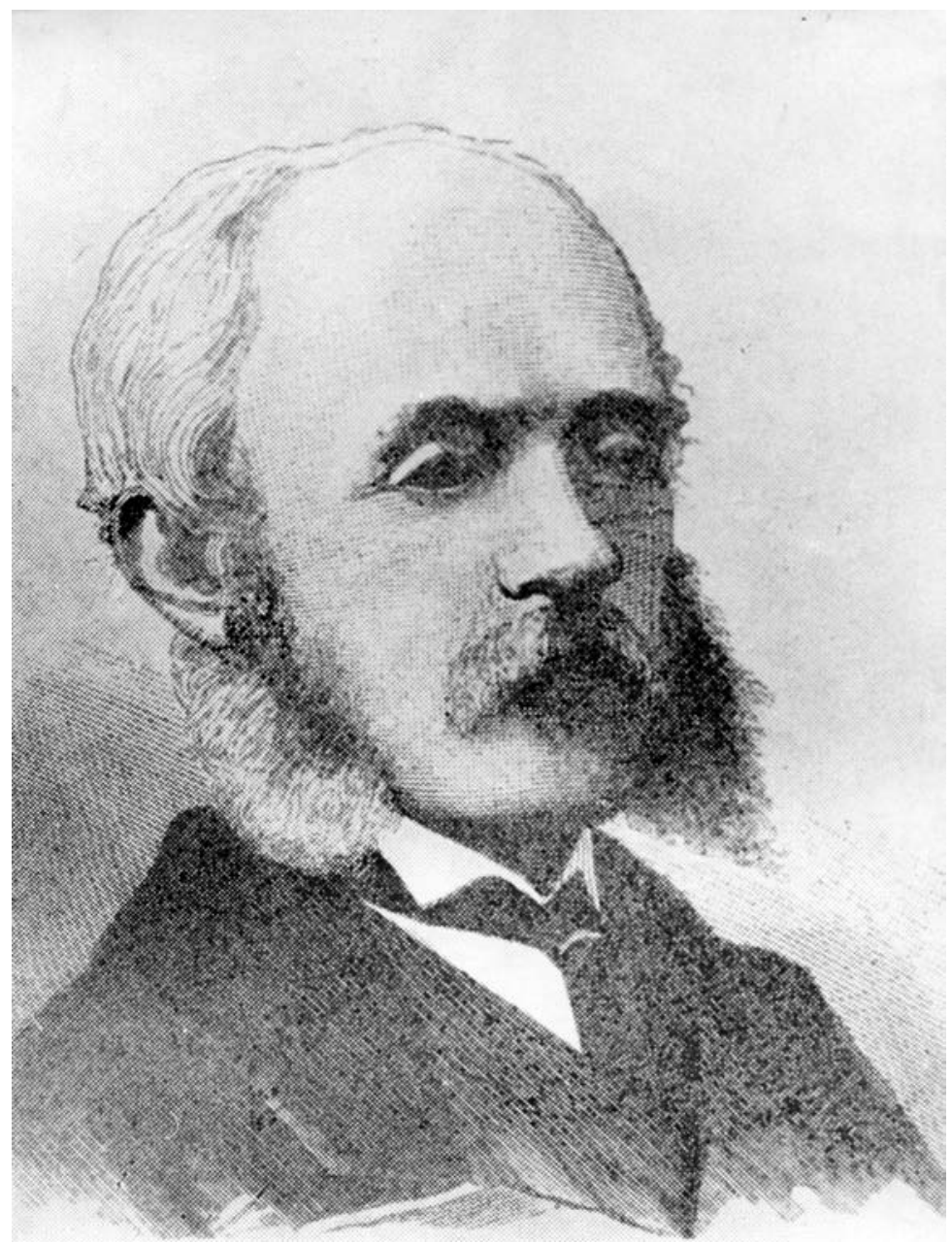

Fig. 1 - Emilio Cornalia (1824-1882). Archivio fotografico MSNM. 
La nomina delle cariche direttive avvenne nel corso delle prime due sedute del $1^{\circ}$ e del 23 dicembre 1858: Robiati fu nominato presidente onorario perpetuo, Cornalia confermato presidente e il naturalista Antonio Villa (1806-1885) vicepresidente. La segreteria fu invece affidata congiuntamente al geologo mineralogista Giovanni Omboni (1829-1910) (Fig. 2) e all'abate Antonio Stoppani (1824-1891), il quale aveva da poco pubblicato i suoi Studii geologici e paleontologici sulla Lombardia. Conservatore e viceconservatore divennero rispettivamente l'ittiologo Cristoforo Bellotti (1823-1919), già conservatore del Museo, e il medico naturalista Gaetano Barzanò (1819-1859).

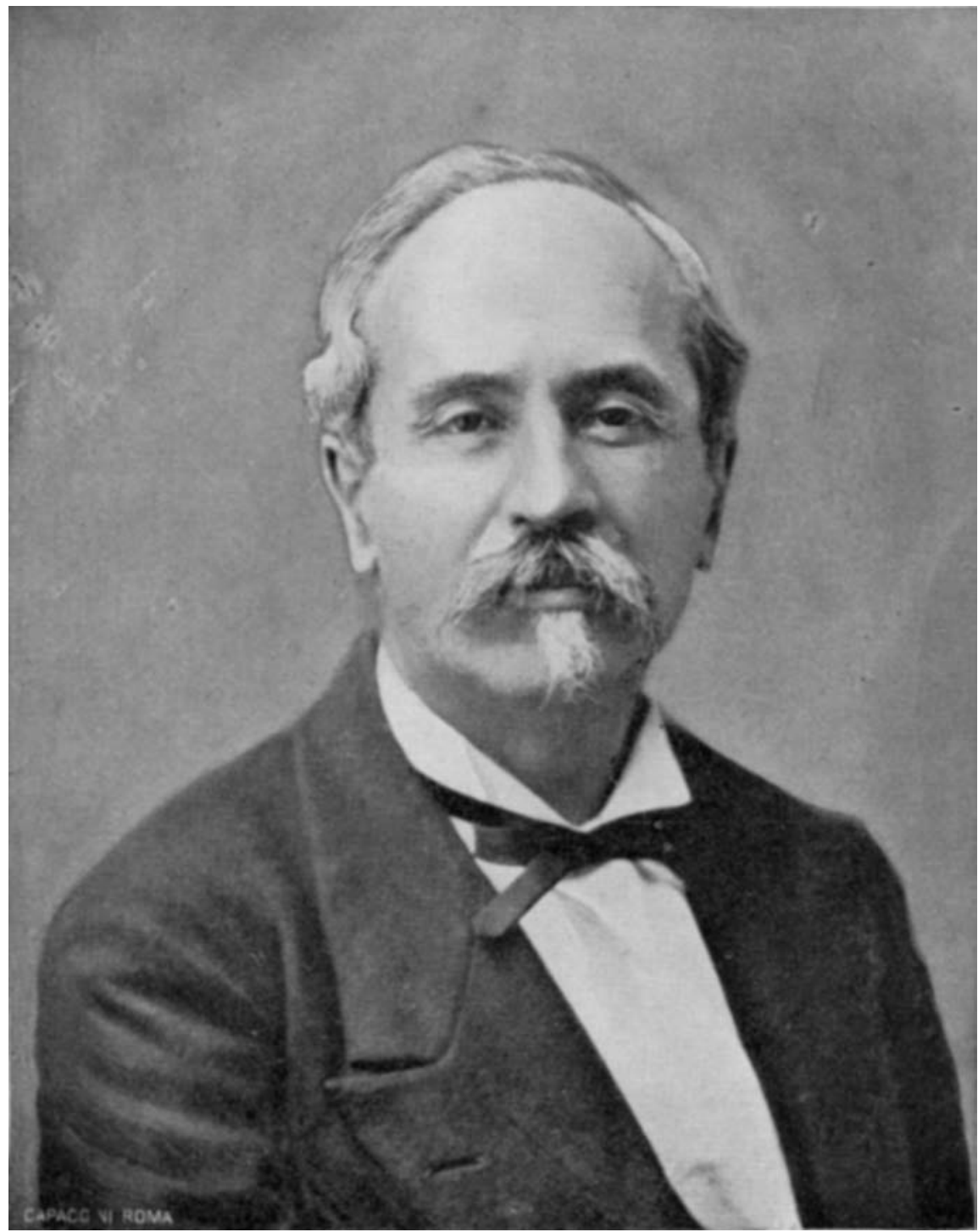

Fig. 2 - Giovanni Omboni (1829-1910). Archivio fotografico MSNM. 
Per quanto riguarda la fisionomia dei soci fondatori, sul totale dei 153 presenti nel 1857, 103 erano residenti a Milano; 21 di loro lavoravano nell'amministrazione statale (in particolare presso la Direzione delle Pubbliche costruzioni, l'Amministrazione del censo, la Giunta del censimento e soprattutto l'I.R. Contabilità di Stato); 28 erano professori di liceo o di scuole specialistiche, 8 professori universitari e 4 impiegati di società ferroviarie. Nello specifico, 30 soci erano ingegneriarchitetti, 18 nobili, 16 docenti di storia naturale o scienze naturali, 14 religiosi, 8 ragionieri, 8 chimici e farmacisti, 6 professori di fisica, 4 medici (ma erano certamente molti di più, in quanto la maggior parte dei naturalisti aveva una laurea in medicina), 4 computisti e un solo avvocato ${ }^{7}$. Complessivamente, dunque, nella fisionomia iniziale della Società ritroviamo non solo una forte impronta milanese $\mathrm{e}$ lombarda, ma anche da un lato l'interesse dello Stato (austriaco) per la conoscenza del territorio e lo sfruttamento delle risorse minerarie, dall'altro l'interesse delle professioni tecniche per i risvolti applicativi della geologia, soprattutto nel settore delle costruzioni.

Il 23 gennaio 1859, il presidente Cornalia propose ai soci il Programma di studii della Società, in cui poneva le basi per il lavoro futuro e allargava il campo degli interessi e degli studi, ad esempio insistendo sulla zoologia, disciplina da lui prediletta, che aveva uno stretto legame con l'agricoltura, con la geologia e con le altre scienze naturali. In particolare, egli esortava a studiare una parte del mondo animale ancora sconosciuta, quella degli invertebrati e degli insetti, che "in Francia e in Germania formano ora lo studio, direi, di moda di molti entomologi"'. Spiegava inoltre quale dovesse essere il nuovo ruolo del naturalista, vero scienziato e studioso della "scienza viva", non più solo collezionista e osservatore distaccato della natura: "Ormai il naturalista non deve esser più semplice raccoglitore; le sue osservazioni non si devono più limitare alla superficie, alla corteccia, direi così, dell'animale; d'un tale naturalista sarebbero sterili le lunghe fatiche, sarebbe il suo un amore, che al minimo ostacolo può raffreddarsi e lasciargli mirare con indifferenza studi ed oggetti che altre volte furono la gioja de' suoi passatempi, il sollazzo delle sue gite campestri, il sollievo fra le sue più serie occupazioni. In ciò dovete trovare il motivo che molti tra noi si mettono agli studj delle scienze naturali e pochi poi perseverano in essi. La scienza viva, che ora di preferenza si coltiva, abbraccia campo più vasto, ama internarsi nell'organismo e studiarne le minime modificazioni; ama vedere la metamorfosi degli organi che reggono alla loro volta la fisiologia dell'animale. Allora solo si riesce ad avere di questo un concetto pieno, completo, conoscendone così i suoi rapporti cogli altri e svelandosi il suo significato nel piano della creazione. Studii così iniziati hanno troppa attrattiva per esser così di leggieri abbandonati. Essi perdono l'aspetto del semplice trastullo, e s'addicono ai severi e filosofici pensamenti dell'età più avanzata"'.

L'utilizzo del microscopio aveva del resto dischiuso un nuovo mondo agli occhi degli scienziati e Cornalia sottolineava l'importanza del "mutuo rapporto"10 tra le scienze, in particolare tra la zoologia e la geologia, tanto che gli "esseri infinitamente piccoli" gli apparivano "più necessarj all'economia del creato [...] che non $\mathrm{i}$ colossi animati" 1 . Questo approccio si addiceva naturalmente al nuovo corso degli studi scientifici, che proprio negli anni cinquanta dell'Ottocento avevano visto lo sviluppo della ricerca di laboratorio, il perfezionamento delle tecniche microscopiche e il progressivo imporsi della biochimica.

Anche il geologo della nuova generazione avrebbe dovuto concentrarsi, piuttosto che sui grandi cataclismi, sulla quotidianità dei mutamenti a cui era soggetta la 
terra e sull'azione costante dei fenomeni atmosferici e meteorologici. La geologia avrebbe dovuto inoltre fornire importanti strumenti all'economia, rivelando la presenza nei terreni di metalli e sostanze utili all'industria e all'agricoltura.

Uno degli scopi della Società, dunque, doveva essere, oltre a quello di realizzare finalmente una carta geologica del Lombardo-Veneto, anche quello di compilare un catalogo delle sostanze minerali e dei fossili presenti nei terreni lombardi, seguendo di fatto le sollecitazioni che arrivavano in questo senso dall'Istituto geologico di Vienna $^{12}$.

\section{La Società Italiana di Scienze Naturali e l'Unità d'Italia}

Ma nel 1859 era ormai tempo di allargare lo sguardo oltre confine: 1'Unità d'Italia era alle porte e i naturalisti milanesi e lombardi sposarono in massa la causa del Risorgimento. Interrotte in aprile le attività sociali per lo scoppio della seconda guerra d'indipendenza dall'Austria, la Società geologica riprese le sue sedute il 21 agosto 1859, in un clima politico completamente rinnovato. La Lombardia si trovava ora annessa al Piemonte, primo tassello di quella che in poco meno di due anni sarebbe diventata l'Italia unita. Cornalia aprì quindi la seduta commemorando la figura del socio Torquato Canetta (1837-1859), giovane medico naturalista arruolatosi volontario nei Cacciatori delle Alpi e ferito a morte 1'8 giugno a Seriate (Bergamo) nello scontro con gli austriaci. Egli aveva iniziato a riordinare la collezione d'insetti del MSNM e - commentava Cornalia - avrebbe certamente portato a termine il lavoro "se le voci d'indipendenza e di guerra non avessero cominciato a serpeggiare fra noi, ed a far battere più forte i nostri cuori". Canetta era stato infatti "il primo a sospendere ogni scientifico lavoro, ed a seguire in vece la questione politica nel suo rapido svolgimento, sempre fisso in mente, che il primo dovere di tutti noi è la difesa e l'indipendenza del proprio paese"13.

Il 20 novembre 1859, poi, l'annessione al Piemonte veniva suggellata in seno alla Società anche con la nomina a socio di Quintino Sella (1827-1884), ingegnere e geologo, professore al Regio Istituto tecnico di Torino, poi ministro delle finanze e fondatore del Club alpino italiano, il quale proprio in quei mesi collaborava con il Ministero della pubblica istruzione alla preparazione di "un progetto di ordinamento dell'Istituto tecnico da crearsi a Milano"14 (il futuro Politecnico) ed era quindi in contatto con l'ambiente tecnico-scientifico milanese.

L'anno 1859 si chiuse con la seduta del 18 dicembre, nella quale si fece un bilancio dei primi anni di attività e si rilevò come, nonostante fossero ormai presenti solo 138 dei 153 soci fondatori originari, la Società fosse in realtà cresciuta, poiché nel solo biennio 1858-59 erano stati nominati ben 42 nuovi soci effettivi.

Ma di fronte ai recenti rivolgimenti politici i naturalisti milanesi sentivano l'esigenza di voltare pagina. L'ultima seduta del 1859 servì dunque per sottolineare, anche nella denominazione sociale, la fine di un'epoca: "Si passa finalmente alla discussione sul come si possa modificare il nome della Società, in vista delle nuove circostanze politiche e della varietà degli studj di cui essa si deve occupare, al fine di indicare col nome che la Società vuole estendere il suo campo d'azione a tutta l'Italia e si occupa di tutte le parti della storia naturale"'15.

Si trattava del resto di un desiderio presente anche in alcuni naturalisti del sud, come si legge in una lettera inviata qualche mese prima a Cornalia dallo zoologo napoletano Oronzio Gabriele Costa (1787-1867), già colpito dai provvedimenti repressivi del Governo borbonico dopo il 1848, quando era stato destituito 
dall'insegnamento universitario e aveva visto chiudere l'Accademia degli aspiranti naturalisti da lui fondata: "Non poteva Ella farmi cosa più grata quanto impiegandomi a contribuire al benessere ed avanzamento di cotesta Società scientifica, di cui meritamente Ella siede a capo. Quantunque reso nullo dall'altrui iniquità, pure sento ancor come prima essere italiano; ed a questo nome sorgo sempre con eguale energia. Eccomi dunque sollecito a rispondere al suo onorevole invito con la voce e con l'opera. [...] I Governi illuminati sentono ora massimamente il bisogno e l'importanza dell'associazione intellettuale. Laonde, oltre le proprie istituzioni, lasciano libero il campo ad altri di potersi assembrare, per coltivare e promuovere col reciproco soccorso le Scienze le Lettere e le Arti d'ogni maniera. [...] Ma sventuratamente le Scienze Naturali qui debbono dormire, perché sono guardate con occhio bieco. La gioventù arde di desiderio di apprendere, ma da chi? coltivarle, e perché? e con quali mezzi? [...] Vorrei essere ricco di mezzi per quanto sento nel cuore, onde far sperimentare a questa novella italiana istituzione gli effetti dell'animo mio"16.

La discussione sul nuovo nome da dare alla Società si concluse il 22 gennaio 1860, quando i soci votarono la denominazione di Società italiana di scienze naturali, volendo sottolineare, oltre al legame con il nuovo territorio italiano, l'intenzione di non restringere il campo degli studi alla sola geologia e di ottenere quindi una maggiore visibilità per la pubblicazione degli Atti, che in questo modo sarebbero stati letti anche "dai non geologi"" .

La nuova dimensione nazionale - per quanto ancora incerta nei suoi confini - portò subito un cambiamento nel Regolamento generale della Società: nella seduta del 24 febbraio 1860 venne infatti decisa l'eliminazione del paragrafo 25, che si riferiva alla presenza di un commissario governativo alle riunioni, simbolo dell'odiata sorveglianza austriaca, mentre al paragrafo 15 i soci corrispondenti non erano più considerati quelli residenti al di fuori del Lombardo-Veneto, bensì al di fuori dei "Regj Stati".

Nei decenni successivi la retorica risorgimentale continuò poi a enfatizzare il ruolo nazionale della SISN e l'impegno patriottico dei suoi promotori, come scriveva il segretario Napoleone Pini in una relazione del 1884: "Solo chi visse in Milano nei tristissimi giorni che succedettero al moto insurrezionale del 6 febbraio 1853, in cui la parola gelava sul labbro per tema del carcere o del capestro, solo colui può immaginare le difficoltà frapposte da quel Governo alla costituzione di questa Società, ed apprezzare in modo condegno l'opera indefessa, sagace, patriottica dei benemeriti suoi fondatori. [...] Dopo il fortunato rivolgimento operatosi nel nostro paese nell'anno 1859, mercé il quale le diverse provincie d'un'unica nazione poterono nel santo nome della libertà stendersi la mano, chiamarsi dal nome della patria loro, desiosa di estendere il suo campo d'azione a tutta la penisola, ed il suo dominio a tutti i rami della scienza che i misteri della natura indaga, studia e rivela, assunse l'attuale denominazione di Società italiana di scienze naturali"'18.

Il 22 aprile 1860, all'indomani dei plebisciti di annessione al Piemonte dei ducati di Modena e Parma, del Granducato di Toscana e delle Legazioni pontificie, Cornalia proponeva alla SISN di occuparsi di alcuni temi interessanti in rapporto alla prosperità industriale ed economica del paese, anche se in realtà lo sguardo era ancora rivolto prevalentemente alla Lombardia: si trattava di studiare da un lato $\mathrm{i}$ rimedi alla malattia del baco da seta che stava distruggendo una delle principali risorse economiche lombarde, dall'altro la piscicoltura, ovvero l'allevamento artificiale dei pesci in appositi bacini e nei laghi di cui abbondava la regione, in par- 
ticolare nel lago del Segrino in Brianza. Quest'ultima proposta fu particolarmente apprezzata dai soci soprattutto per i suoi risvolti sociali: il nobile imprenditore Carlo Tinelli (1795-1877) affermava infatti che in seguito all'impoverimento ittico dei laghi lombardi si finiva per sottrarre "singolarmente alla classe povera, un alimento che fu sperimentato eccellente in unione cogli alimenti tratti dai cereali, ai quali i poveri sono ormai quasi esclusivamente ridotti" ${ }^{19}$.

Il tema politico, tuttavia, era sempre presente in quei mesi convulsi di grandi cambiamenti: nella seduta del 22 luglio 1860 Cornalia commemorava il socio defunto Abramo Massalongo (1824-1860), naturalista di Verona laureato in medicina, morto il 25 maggio a soli trentasei anni, figura di fervente patriota del quale ricordava gli erbari inviati agli amici lichenologi fuori dall'Italia "in cassette foderate di bianco, listellate di verde, con cartellini in rosso" e per questo sequestrati dal governo austriaco. Massalongo inoltre, morendo, aveva lasciato un testamento in cui raccomandava ai figli di offrire in vendita la sua collezione "avanti tutti al Re italiano, al Re nostro, e in nessun caso mai a sito ove dominasse un principe di Casa d'Austria"'20.

\section{L'epoca d'oro dei congressi}

La libertà di movimento e d'associazione conquistata dopo la partenza degli austriaci consentì alla SISN di partecipare più liberamente ai congressi internazionali e di cominciare a valutare l'opportunità di organizzare convegni propri nel nuovo ambito territoriale italiano.

Il 25 novembre 1860 il segretario Omboni relazionava i soci sul Congresso dei naturalisti svizzeri tenutosi a Lugano in settembre. Si trattava dell'adunanza generale annuale della Società elvetica di scienze naturali, che univa diverse società scientifiche svizzere con sede nelle maggiori città dei cantoni. L'ultima riunione si era tenuta nell'autunno del 1858 a Berna, mentre la successiva, fissata a Lugano, era stata rimandata a causa della guerra d'indipendenza italiana ed era stato possibile ripristinarla solo nel 1860. Se l'afflusso degli studiosi d'oltralpe fu tuttavia scarso, soprattutto per le "piogge continue, che incagliarono straordinariamente i viaggi alpini" 21 e per il contemporaneo svolgimento di altre riunioni in città diverse, la partecipazione degli studiosi dell'Italia settentrionale, in particolare di Milano, fu invece straordinaria e contribuì a dare all'evento la caratura di "un vero congresso scientifico internazionale" 22 . La SISN era rappresentata da tutte le cariche direttive (il presidente Cornalia, il vicepresidente Antonio Villa e i segretari Omboni e Stoppani), nonché dal fondatore Robiati e dai soci Giuseppe Balsamo Crivelli, Filippo De Filippi e Paolo Panceri. Non mancavano poi soci meno conosciuti come il padre Gallicano Bertazzi di Milano e il professor Martino Anzi di Como, mentre tra coloro che si associarono in seguito alla SISN erano presenti il delegato dell'Accademia fisio-medico-statistica di Milano, avvocato Pier Ambrogio Curti, e il delegato dell'Industria commerciale e preparati chimici in Milano, Angelo Bollini.

Il congresso di Lugano si rivelò soprattutto un momento di confronto e di scambio tra studiosi, dal momento che - commentava Omboni - "gli intervenuti, anche perché tenuti insieme quasi per forza dal cattivo tempo e dalla mancanza d'ogni distrazione, ebbero campo di distribuirsi in crocchi diversi, a seconda degli studj speciali d'ognuno, e di discutere famigliarmente di molti e molti argomenti; e poterono così, oltre che riannodare e continuare le antiche relazioni, farne di nuove, e sciogliere o almeno mettersi in casi di chiarire a viva voce molte quistioni, e 
togliere così molte divergenze d'opinione, con molta maggiore facilità e prontezza che colle dispute in seduta regolare e colle Memorie stampate. E questo, forse più che altro, è il miglior risultato dei congressi scientifici"23.

La necessità di organizzare congressi per naturalisti italiani divenne da allora per la SISN una vera priorità. La Società, divenuta ormai "italiana", assunse infatti più consapevolmente il suo ruolo di aggregazione nazionale, rivolgendo lo sguardo al nuovo territorio derivato dall'unione degli antichi stati preunitari. Seguendo ciò che Maria Pia Casalena osserva nel suo lavoro comparativo sui congressi degli scienziati tenutisi in Francia dal 1833 e in Italia dal $1839^{24}$, possiamo dire in sostanza che la SISN, nata su sollecitazione del governo austriaco e incline quindi inizialmente a coltivare la scienza per lo Stato, si tramutò ben presto in un organismo votato alla scienza per la Nazione.

Questa vocazione si manifestò appunto attraverso le riunioni straordinarie fuori sede, veri congressi itineranti annuali organizzati a partire dal 1864 in varie città italiane, grazie ai quali la SISN si propose di studiare il territorio italiano, ancora in gran parte inesplorato dal punto di vista geologico e naturalistico; di legittimare la figura del naturalista come scienziato professionista, quando ancora la sua attività nell'immaginario popolare somigliava più che altro a quella di un curioso e bizzarro collezionista di oggetti naturali; di divulgare la scienza facendone uno dei pilastri del progresso dell'Italia unita; di costituire un centro di riferimento per tutti i naturalisti italiani offrendo loro la possibilità di conoscersi e confrontarsi.

Fin dall'inizio, nel Regolamento generale era stata prevista la possibilità di organizzare "escursioni scientifiche" e "riunioni straordinarie" 25 . Nella seduta del 28 luglio 1861, a pochi mesi dalla proclamazione del nuovo Regno d'Italia, il padre barnabita Michelangelo Manzi di Monza propose di organizzare una riunione straordinaria in occasione della prima Esposizione nazionale di Firenze, ma solo nel caso in cui non si fosse organizzato alcun altro congresso scientifico ${ }^{26}$. Nonostante il favore con cui venne accolta la proposta in ambito fiorentino, la riunione non ebbe luogo, poiché, come annunciava ai soci il presidente Cornalia nella seduta del 24 novembre 1861, "giunta in quella città, la Presidenza trovò tutti i dotti là convenuti, compresi gli stessi Fiorentini, talmente occupati per i giurì della Esposizione, per le riunioni di diverse commissioni, per le sedute del Congresso straordinario scientifico, ecc., che non poté avviare alcuna pratica per attuare la riunione della Società. Terminati i lavori dei giurì, terminate le sedute per le commissioni e il Congresso, e terminate le altre occupazioni, quasi tutti i dotti partirono in brevissimo tempo, e dovettero pure partire anche i socj componenti la Presidenza; e così divenne affatto impossibile la riunione della Società" 27.

L'idea di organizzare delle riunioni straordinarie che rinsaldassero i legami tra $i$ naturalisti italiani non fu comunque abbandonata dalla SISN, la quale contribuì in quegli anni - insieme al MSNM - a fare di Milano un centro di riferimento, tanto che il 3 marzo 1863 Louis Pillet (1819-1894), membro e poi presidente dell'Académie des sciences, belles-lettres et arts de Savoie, scriveva a Cornalia: "Milan tend à devenir la capitale intellectuelle de l'Italie"28.

Finalmente il 31 gennaio 1864 la presidenza inviò ai soci una circolare a stampa in cui dichiarava di aver accettato "l'incarico di promuovere e condurre in atto quelle sedute straordinarie od escursioni scientifiche, dalle quali, in modo però affatto indeterminato, parlano gli articoli 23 e 24 del Regolamento generale" 29 . Cornalia ricordava il rapido sviluppo della Società "dopo i felici eventi del 1859, per cui essa può dirsi veramente italiana, contando numerosi i suoi membri in tutte 
le parti della Penisola"30, il che rendeva ormai "non solo possibile, ma veramente necessario uno sviluppo del primitivo programma, nel quale siano comprese, non già semplici escursioni, ma vere adunanze straordinarie, fuori dell'ordinaria residenza della Società, come è costume delle Società più illustri e più attive, quali la Società geologica di Francia e la Società elvetica di scienze naturali" ${ }^{\prime 31}$.

Gli esempi delle riunioni itineranti delle società svizzere e francesi erano per Cornalia i mezzi più efficaci per raggiungere lo scopo di ogni società scientifica: "promuovere la scienza e dilatarne ad un maggior numero possibile i beneficj" 32 . E in tale prospettiva il presidente elencava alcuni "fini speciali" a cui avrebbe dovuto tendere la SISN da quel momento in poi:

" $1{ }^{\circ}$ Aggiungere stimoli e facilitare l'occasione ai socj lontani dall'ordinaria residenza della Società di trovarsi insieme almeno alcuni giorni dell'anno, sicché abbia luogo, col mutuo scambio degli affetti, quello delle idee, onde sia più rapidamente effettuata, per rapporto alle idee ed al linguaggio scientifico, quella unificazione di cui sente bisogno l'Italia scientifica del pari dell'Italia politica.

$2^{\circ}$ Popolarizzare la scienza, costringendola, se fa d'uopo, ad uscire da' suoi centri naturali, dalle grandi città, per mostrarsi benefica, festiva, popolare ovunque, anche ne' più ermi recessi delle Alpi e dell'Appennino. È un bisogno dell'epoca, è un atto di giustizia a cui soddisfece, con mirabile risultato, la Società elvetica.

$3^{\circ}$ Conoscere il nostro paese, cui dobbiamo confessarci, per molte parti e per troppi lati, il più sconosciuto a noi stessi.

$4^{\circ}$ Rendere agli scienziati stranieri quel tributo di fratellevole accoglienza, che ottennero sempre da loro gli scienziati italiani, e nello stesso tempo soddisfare il giusto e nobile desiderio di far conoscere al di fuori quanto si faccia per la scienza in Italia" 33 .

Il richiamo continuo all'esempio della Società elvetica di scienze naturali non era casuale. Si trattava infatti della "prima accademia votata all'autorappresentazione itinerante", la prima che si proponesse "di radicarsi sull'intero territorio nazionale e di attingere la propria membership da ogni punto di esso" 34 . La Società elvetica intendeva "aggregare attorno alle poche decine di specialisti folle di amatori e ammiratori", ("popolarizzare la scienza", secondo le parole dello stesso Cornalia) e aveva una finalità politica molto simile a quella che muoveva nei suoi primi passi la SISN: ricomporre, tramite i congressi, le discordie tra i cantoni (nel caso italiano tra le diverse entità territoriali), imponendo i valori di quanti lavoravano per il benessere collettivo, gli scienziati, che tendevano ormai ad affermarsi come vere e proprie guide intellettuali del paese. "La scienza aspirava a diventare la nuova religione nazionale" 35 .

A pochissimi anni dall'unificazione, dunque, Cornalia chiedeva ai soci di dichiararsi o meno favorevoli alle riunioni straordinarie e di sottoporre alla presidenza "quei riflessi, o progetti, o proposte che ciascuno credesse di partecipare"36. Tra le prime risposte arrivate, quella del celebre botanico Théodore Caruel (1830-1898) suggeriva, per la buona riuscita dei congressi, la scelta di una sede "in prossimità di luoghi utili a visitarsi da' naturalisti di ogni genere, zoologi, botanici, geologi, affinché alle adunanze accademiche potessero essere accoppiate gite d'istruzione", dando la preferenza a "una città piccola piuttosto che una primaria, purché fosse grande abbastanza da porgere facile accomodamento ai ricorrenti" " Caruel suggeriva poi alla SISN, che aveva sede nel nord Italia, di spostarsi verso il centro o il sud del paese, ad esempio a Sarzana o nei suoi dintorni, in modo da permettere ai congressisti "la perlustrazione delle Alpi apuane come oggetto principale in vista" 38 . 
Di parere opposto, invece, il socio corrispondente duca Federico Lancia di Brolo (1824-1883), agronomo siciliano, che lungi dal preferire "gli ermi recessi dell'Alpi o dell'Apennino", dava la sua preferenza senz'altro alle "grandi città, ricetti di collezioni, di gabinetti, di naturalisti", dove vi era maggiore possibilità di apprendere, di radunare il pubblico, di dare alle riunioni un carattere "più solenne", di avere a disposizione "più giornali" e "apparecchi che non potrebbero aversi in remoti o isolati luoghi". La sede dei congressi avrebbe dovuto essere poi scelta "a giro fra le varie regioni della Penisola, almeno fra le continentali, onde dare un carattere verace di italianità alla Società e al congresso" 39 . Inoltre, dal momento che si trattava pur sempre di una riunione generale della SISN, era necessario, secondo Lancia di Brolo, discutere anche degli affari societari, contribuendo così a dare un "marchio di italianità e di nazionalità all'istituzione, che in ogni altro caso resterebbe esclusivamente in fatto milanese" 40 . Infine, riprendendo una proposta già avanzata nel congresso di Firenze del 1861, il duca sosteneva la necessità di affiancare questa riunione ad altre, al fine di "analizzare meglio il momento intellettuale del tempo e del paese" e di sostituire - come ormai accadeva in Francia - i congressi generali con quelli specialistici: "Quelli servivano agli animi, questi giovano meglio al sapere; quelli potevano riuscir meglio in altri tempi, questi son più adatti al presente; quelli hanno più solennità e pubblicità, questi son più gravidi di risultanze" 41 .

Se quindi i grandi congressi degli scienziati del recente passato avevano avuto prevalentemente la funzione di infiammare gli animi dei patrioti rinsaldando $\mathrm{i}$ legami nazionali tra gli studiosi dei vari stati della penisola, indipendentemente dal loro settore disciplinare, all'indomani dell'Unità erano le società scientifiche specialistiche a farsi carico del sentimento nazionale, mentre il naturalista acquisiva una nuova professionalità e la scienza stessa diveniva sempre più "popolare".

Del resto in quello stesso agosto 1864 anche il presidente della Società agraria di Lombardia (fondata nel 1862) invitava i soci della SISN al Congresso e alla mostra d'agricoltura che si sarebbe tenuta in settembre a Pavia, "pel vivo desiderio di più tenacemente rannodare $\mathrm{i}$ vincoli di quella nazionale fratellanza che dai Congressi scientifici riconosce il suo primo iniziamento" 42 .

La questione della divulgazione scientifica appariva in questo contesto assolutamente centrale. A tale proposito Antonio Stoppani, di ritorno dal Congresso dei naturalisti svizzeri tenutosi a Samaden nell'agosto 1863, riferiva meravigliato ai colleghi della SISN che durante il tragitto dalla Valtellina attraverso il passo del Bernina era stato più volte interrogato dagli abitanti del luogo in un modo inusuale, che rivelava a suo parere una nuova percezione popolare dei congressi scientifici. Scriveva infatti: "La parola d'ordine, l'imprescrittibile domanda, fosse in italiano, in francese, in tedesco, era questa: va alle feste? Questo modo così singolare, così nuovo per esprimere il concetto di un congresso scientifico mi ha lasciato, vi ripeto, un'impressione assai profonda. Questo modo, passato quasi per incanto nelle convenzioni del linguaggio, adottato senza sottigliezze, affatto naturalmente dal popolo, il cui linguaggio è sempre così filosofico quando esprime l'effetto che producono nell'animo gli esterni avvenimenti, vi dice quanto debba esser divenuto colà popolare il concetto della scienza, quanto graditi, preziosi, festeggiati il nome e la persona dello scienziato. Vi assicuro che non mi aspettava un tale progresso nell'ordine delle idee popolari, su quei gioghi inaccessi, tra quei ghiacci eterni, in paesi isolati quasi per otto mesi dal consorzio del mondo"43. Uno scienziato straniero, anch'egli stupito da "tanta popolarità guadagnata dalla scienza", gli 
aveva allora domandato: "quando credete voi si potrà combinare una tal festa in Italia? ditemi, fra dieci anni?". Era dunque comune l'idea che l'Italia fosse ancora indietro nel campo della divulgazione scientifica, ma Stoppani replicava fiducioso: "la Società italiana di scienze naturali saprà rispondere col fatto, meglio che colle parole al nostro amico d'oltralpe" 44 .

Fu nel 1864 che la SISN si diede ad organizzare il suo primo congresso nazionale, la sua prima riunione straordinaria fuori sede. La città prescelta fu Biella, residenza dell'illustre socio Quintino Sella, che contribuì a organizzare l'evento e ne tenne la presidenza. I lavori si svolsero regolarmente dal 3 al 6 settembre, anche grazie alla segretaria generale affidata all'abile segretario della SISN Giovanni Omboni.

Nel suo discorso d'apertura, Sella dichiarava che il modello seguito dalla SISN era ancora una volta quello della Società elvetica di scienze naturali, che aveva introdotto i congressi fin dal 1815. In Svizzera, del resto, "l'assenza di un centro, di una capitale che assorb[isse] la migliore attività degli ingegni di una vera nazione", aveva fatto "pria che in ogni altro luogo sentire il bisogno di riunioni di questa natura"45. Tutti i maggiori paesi europei, dall'Inghilterra alla Francia, dalla Germania alla Scandinavia, ne avevano ben presto seguito le orme. Ma anche l'Italia - proseguiva - prima dell'Unità aveva conosciuto i congressi degli scienziati, quando il paese era ancora suddiviso "in tanti regnucci in miniatura, i cui governi per la natura stessa delle cose dovevano essere ed erano, come tutti i deboli, pieni di sospetti e di diffidenze". In quel tempo i patrioti si erano ingegnati "più o meno sottilmente di affratellarsi, di darsi a conoscere a vicenda, di far penetrare nelle masse il concetto, che l'Italia, una per natura e per diritto, doveva anche essere una di fatto". Così gli scienziati avevano avuto "la felicissima ispirazione di iniziare congressi puramente scientifici, che potessero sfuggire alle ombrose paure dei governi"46. Ma dopo l'Unità - continuava Sella - la "ragion politica" di quei congressi "era venuta meno" ed era apparso chiaro che la loro natura generalista non era funzionale a tutte le discipline, per le quali occorrevano ora congressi di tipo specialistico ${ }^{47}$.

Sella passava poi a dichiarare che era necessario "dare all'Italia la sua carta geologica in grande scala", proponendo che ogni città designata a sede di una riunione straordinaria della SISN presentasse al congresso la carta del proprio circondario. Con ciò egli cercava di fare ammenda della decisione presa nel 1862 come ministro delle finanze (ricordato e odiato per le sue "economie fino all'osso"), di ritirare i finanziamenti alla direzione della carta geologica d'Italia, ch'egli stesso aveva istituito pochi mesi prima: "E se questo esempio nostro fosse seguito parrebbe, a chi ha l'onore di parlarvi, di avere intieramente riparato ad una specie di infanticidio, di cui credette suo debito di rendersi colpevole, allorquando, per riguardo alle imperiose strettezze finanziarie del nostro paese, ebbe ad ordinare nel 1862, che si sospendesse quel decreto del $1861^{48}$, intorno a cui egli aveva avuta tanta parte, e che ordinava la formazione a spese del governo della carta geologica d'Italia alla scala da 1 a 50.000" 49 .

Sella proseguì quindi illustrando la costituzione geologica e le condizioni economiche del circondario di Biella, mentre il presidente Cornalia - che insieme al socio Filippo De Filippi aveva trovato ospitalità nella cittadina piemontese presso l'ex podestà di Milano, il senatore Gabrio Casati ${ }^{50}$ - tenne invece una relazione Sull'origine e sullo sviluppo della Società italiana di scienze naturali, un'occasione per rileggere gli anni di fondazione alla luce dell'epopea risorgimentale: "L'Italia, 
più ancora che in tanti scompartimenti territoriali, era intellettualmente divisa in molti e piccoli centri, che, isolati, vivevano estranei fra loro senza quella vita che l'attrito solo e le mutue relazioni sono capaci di far sviluppare. [...] Ma frattanto i tempi maturavansi e l'Italia guidata dal suo buon genio spezzava le barriere che la dividevano in mille parti, e colla sôrta aurora politica sorgeva un miglior avvenire alla Società nostra. Torino, Parma, Modena, Bologna, Ancona, Ascoli, Napoli e ben altre città sorelle corrisposero al pressoso nostro invito e i soci crebbero in ogni parte del suolo d'Italia, non solo volonterosi contribuenti, ma soci, attivi e benemeriti cultori de' nostri studi, soci, direi, militanti della scienza. Le forze della penisola concorsero riunendosi in una comune rappresentanza delle nostre scienze come era nostro desiderio e nostra speranza" 51 .

Nella nascente mitologia del Risorgimento, lo scienziato acquisiva una nuova veste eroica, usciva dal suo laboratorio per combattere l'oscurantismo, era riconosciuto dalla popolazione e veniva paragonato a un vero e proprio "soldato della scienza" al servizio della patria: "Egli è tempo che il zoologo che suda nello studio di un piccolo organismo, cui per conoscere arma l'occhio di poderosa lente; il geologo che s'arrampica sui monti per studiarne la struttura e strapparne il marchio della loro età, non sia più oggetto di attonita e indiscreta meraviglia, o tenuto quasi per persona di poco senno. Egli è tempo che tutti riconoscano il beneficio de' nostri studj, e come questi concorrano al progresso ed all'onore della nazione. I nostri fratelli di Svizzera non sdegnarono, or è un anno, di raccogliersi fra le balze coperte d'abeti nella più elevata parte dell'Engadina, ove quei semplici valligiani fecero plauso ai nuovi soldati della scienza, le cui vittorie sulla Verità aggiungono rispetto alla bandiera della loro patria" 52 .

E facendo riferimento all'unificazione della penisola, cui mancavano ancora le terre del Veneto e del Lazio, Cornalia ribadiva: "Questa terra ormai non è più un nome geografico ma una nazione potente che si prepara alla sua finale unificazione, alla sua totale indipendenza. Chi si prepara colla spada, chi ponderando leggi, noi coi progressi della scienza" ${ }^{53}$.

Occorreva rinnovare, a quel punto, anche gli scopi della SISN, di cui egli teneva a sottolineare la differenza rispetto alle antiche accademie: "Poche le forme, nessune le etichette proprie alle vecchie accademie d'altri tempi, noi accogliamo quanti cercano il progresso, siano pur di que' volonterosi che le prime orme cercano stampare sulla via della scienza" ${ }^{4}$.

La libertà necessaria al nuovo associazionismo scientifico postunitario implicava dunque, secondo Cornalia, il coinvolgimento di tutti i naturalisti, indipendentemente dalla loro appartenenza istituzionale, compreso quel folto gruppo di "amatori" che con il loro impegno quotidiano potevano aggiungere piccoli tasselli all'insieme delle conoscenze scientifiche di cui aveva bisogno il paese. Era inoltre finito il tempo in cui bisognava "ricorrere a straniere fonti per conoscere la storia naturale d'Italia": ora il territorio italiano poteva finalmente essere studiato dai naturalisti italiani. L'isolamento degli scienziati prima dell'Unità e "i pochi mezzi di cui potevano disporre" aveva infatti impedito troppo a lungo all'Italia "di partecipare a quella febbre, direi, di scienza che invase in questi ultimi tempi le più colte nazioni d'Europa" 55 .

L'indipendenza politica conquistata imponeva che la scienza non fosse più elitaria, che uscisse dai laboratori, dalle accademie e dalle università per diventare patrimonio comune a tutti i cittadini. "Popolarizzare la scienza" divenne allora una parola d'ordine anche per la SISN e per i naturalisti del Museo milanese ad essa 
collegati: "Riunirsi, consultarsi, mutualmente aiutarsi, produrre, popolarizzare la scienza: ecco la missione di questa nuova Associazione i cui membri attestano qui colla loro presenza quanto siano concordi nel nobilissimo scopo"

Anche la scelta dei centri minori come sede dei congressi rientrava in parte in quest'ottica divulgativa: se i grandi congressi del passato esigevano "i grandi e popolosi centri", le nuove riunioni scientifiche specialistiche preferivano $i$ "siti minori" che fornissero "materia a scientifiche discussioni" e consentissero di non perdere di vista "la missione nostra, quella di rendere popolare la scienza" 57 .

Il primo congresso della SISN a Biella, con le sue tre sezioni di geologia, zoologia e botanica, si concluse con un pieno successo: "L'esito fu soddisfacente oltre quanto si poteva sperare, perché numeroso il concorso, animate le sedute, copiosi e importanti i lavori presentati alle diverse sezioni" ${ }^{58}$. La Società acquisì in quei pochi giorni 13 nuovi soci, tra cui alcuni studiosi eccellenti come Eusebio Oehl, professore di fisiologia all'Università di Pavia, il barone Vincenzo Cesati di Vercelli e Moritz Schiff, professore di fisiologia al Museo di Storia Naturale di Firenze. Nei mesi successivi le adesioni si moltiplicarono e anche i giornali diedero spazio all'evento, come ad esempio il "Giornale dell'ingegnere-architetto ed agronomo", che definì la riunione "brillantissima" 59 .

Sull'onda del successo, l'anno seguente la riunione annuale straordinaria fu organizzata dal 18 al 21 settembre 1865 a La Spezia (Fig. 3), sotto la presidenza di Giovanni Capellini, professore di geologia all'Università di Bologna. In realtà la presidenza era stata inizialmente offerta al marchese Giacomo Doria (18401913), che aveva rifiutato per i numerosi impegni, in seconda battuta al geologo marchese Lorenzo Pareto (1800-1865), che però si era ammalato ed era morto poco dopo, e solo in terza battuta a Capellini. La segreteria era stata invece affidata ancora una volta a Giovanni Omboni, vero braccio destro di Cornalia nella gestione della SISN.

Il discorso d'apertura di Capellini, come voleva il regolamento delle riunioni straordinarie ${ }^{60}$, fu dedicato alla storia naturale del Golfo della Spezia e dei suoi dintorni. Cornalia ribadì in parte le cose dette nel congresso precedente sulla storia della Società, "per mostrare come essa possa avere molta influenza sul progresso della scienza e sull'unificazione scientifica e politica dell'Italia"61.

Una delle novità fu invece l'istituzione, da parte della SISN, di un Congresso paletnologico internazionale annuale che riunisse "tutti gli uomini che si occupano di ricerche preistoriche" 62 . Come prima sede del nuovo congresso, che si sarebbe tenuto nell'agosto 1866 sotto la presidenza del geologo Edouard Desor (18111882), fu scelta Neuchâtel, in Svizzera, mentre il secondo si sarebbe svolto a Parigi, in occasione dell'Esposizione universale del 1867. Ricordando quegli anni molto tempo dopo, lo studioso Pompeo Castelfranco (1843-1921) scriveva: "Quel periodo di anni fu per la Società nostra un periodo di floridezza e di attività. La scienza nuova, giovane e ardita, veniva a dare una nuova vita alle nostre riunioni, e ne animava le discussioni. Da ogni parte affluirono i soci. Ogni più piccolo proprietario di torbiera, nella quale si fosse rinvenuto per avventura qualche coccio di stoviglia o qualche selce lavorata dall'uomo, si sentì paletnologo e chiese di far parte della nostra Società; e siccome pareva allora che bastasse saper distinguere una freccia di pietra da una fusaiola di terra cotta per proclamarsi paletnologi, e annunciarne la scoperta con quattro linee di scritto, la paletnologia diventò di moda; tutti si occuparono di paletnologia, e gli anni 1864-1866 furono tra i più prodighi di scoperte paletnografiche più o meno importanti e di pubblicazioni analoghe" ${ }^{\prime 3}$. 
. (Pubblicazione Straordimaria)

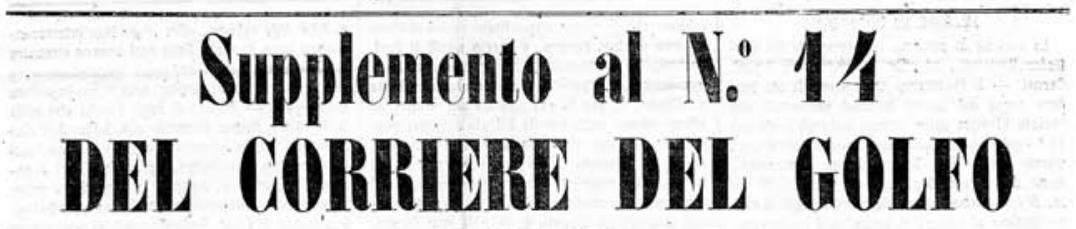

IN OCCASIONE

DRLL SECOIDI RIIIIOIR STRLORDIIIRII DELL SOAETI ITILIII

DI SGIENZE NATURALI

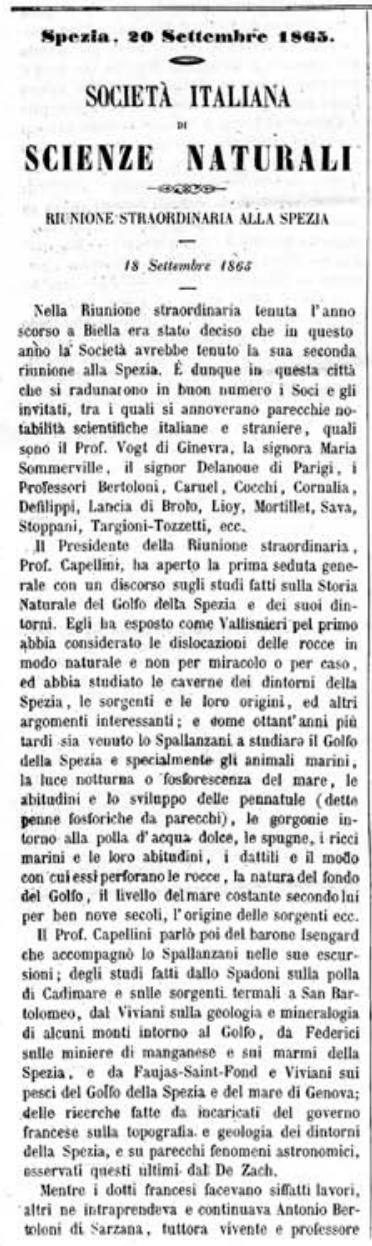

a Bologna. Siffatti lavori si riferivano particolarmente al regoo vegetale. Nello stesso tempo Gerolamo Guidoni di Vernamz cominciò ad oceuparsi della geologia dei dintorni del Golfo, pubblicando
delle osservazioni interessantissime. Poco dope cominciò una lunga serie di studi fatti dal Prof Paolo Savi, dal De la Béche, dal lloffmann, dal Pilla, dal Pareto, dai professori Sismonda, Co quand, Delanoue, Murchison, Meneghini, Cocch e Capellini: osi che orà si puob dire essere i dintorni del Golfo della Spezis una delle regioni geoloricamentc meglio note in Italis I prodott del Begne anima e del resetale funcono studiti

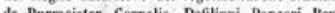
da Burmeister, Cornalia, Defilippi, Panceri, Bertoloni, Caldesi, Rosellini, ecc. GT insetti furonc articolarmente studiati dal Mre Giacomo Doria Considerando tatti questi stadi finora fatti, Prof. Capelliní espresse il sao voto che la Societ abbia a far si che, per mezzo di speciali monografle da essa pubblicate, venga presto a compiersi cio che ancora manca alla completa conoscenza di questo paese interessantissimo per I sua costitnzione geologica e per i suoi prodoti animali e vegetali, cosi come uoo dei più helli per le forme dei moati e per la purezza der cielo. - E la Spezia, conchiuse il Prof. Capellini, raminenterà sempre con orgoglio che i Saturalisti Italiani ani raccolti nel 1865 decretarano onera, da far tacere una volta chi tromosorente opera, da fir tacre a volta cai troppo sorente Fivermenteciaccasa di acgligenza e di inerzia Prailo il discorso del Prof. Capelini, il Segre. tario Omban indico pareechi libri presentati is

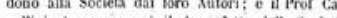
pellini stesso accentó if doto fatto dalla societ al Municipio della Spezia, di un esemplare dei suoi Atat a delle sue Nemorie finora pubblicate. Al che rispose it $\mathrm{K}$. Delegato Straordinario per Ia Citla di Spezia (Presidente oborario della fita. nione), ringraziando in nome dells Citta da Ini ora anministrats.

II signor Delanone, invitato dal Presidente. disse defla origine dei filoni irregolari e metal liferi per mazzo dell' axione di sorgenti minerali con eloruri trollati metallici sulle rocce calearee attraversate da quelle acque.

II Presidente erdinario dellh Societa, Prolessor Emilio Conalia ba parlate dell'origine dell Enciets fondata

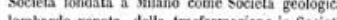
Tomardo resta, de trastormazione in Sociec Italiana di Scienze Natarali, det suo crescente sviluppo. e delle speranze che si hanno sul suo avienire. Ma per quaesto, disse egli, bisogna che la Societs acquisti membiri di tutte le pari d'Italia, e pubblichi memorie mandate da quest Soci: e gia ta Società è andata ogni anno allmentando le sue pubblicazioni, con memorie mandate da Soci residenti non solo in Loonbardia, ma anche in Piemonte, nel Veneto, nell' Italia centrale, a Napoli, a Messina, cece, e la cominciato a fare queste rinnioni straordinarie allo scopo di ravvicinare i dotti dei diversi paesi ditalia, edi far meglio conoscre la Socieis, e raccogliere sempre nuovi membri. Cosi aumentando it numero dei suoi componesti, e le su pabhlicazioni, e facendo conoscere sempre pít i snoi scopi, la Societa sarà sempre pih̀ ntile al progresso della storia naturale in Italia, e alli sempre miglior conoscenza del nostro paese. alla sempre maggior unificazione scientifica politica degfi Italian

Dopo il discorso del Presid. ordinario Corrialia furono acclamati Soci corricpondenti la signera Maria Sommervilte e il signor Mturchiton dilondra La seduta fil chines eon delle parole inumervisate dat Prof Tangion-Tonzelli, parole impirorvisate dal Prof. Targouni-Tozzetti, it quale dissdella pecessita che alle sommith scientificlie gi cistenti in Italia vengano ad aggregarsi moli

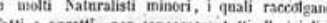
fati e oggetti, per concorrere futti alla highlior conoscenza del paeste, o at progresso della cienzs; 6 disse che questo tinira per essere if risultato delle riunióni straordinarie e delle pabblicazioni đella Societi. Egli espresso anche it desiderio che venga un tempo, nel quale it naturalista non sia piú per $i$ profani nn essere strano, qualche cosa come una specie particolar. del genere nomo, moggetto disempliee curiosit e di riso per il volgo, ma tutti, grandi a pic coli, dotti a indotti, tutti oli laliani conosean a che tendone e che fanto $i$ naturalisti, e ren-

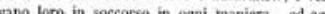
gancluse

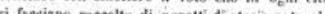

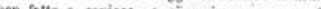

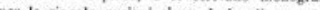
per le singole regtoni e per i singoli gruppi a oggeti, in modo che st abbiano alla fine suticienti materiali per fare una descrizione ben completa dell' thalia e dei saoi prodotti d'ogni. specie. Có cui effi spera di vedere avverato in un tempo non lontano, considerando il mode. affettuoso e cordiale con cui la cittá della Spezi ha accolto i Natufalisti qui accorsi in quest" anne e il buon numero di nersone accorse alta pubblica sedata

Dopo la seduta ci fu il pranzo Sociale, e dopequesto i Mernbri della Rianione si rocarone fun aleuni canolli rimorchiati da due vaporini, gentilema squisita dell" Ammiragliate) allo sces

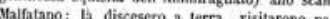
recechi strati fossilieri alla pora disitarono pa-

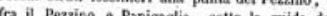
ira il Peczino e Panigaglia, sotto la guda de Prot. Capelini; eda Panigaglia rientrarono nei ca. notti, e ritornarono alla Spezia, che troraronefestosamente illuminata e rallegrata dalla musios della Guardia Kazionale.

19 Settembire 186s: -

In questo giorno si tennero le sedinte speciali delle serioni di Zoologia, Botorica e Geologia Mincrologin, in cai si sono distribuiti i membri della Rinnione per la trathatione dei particolaris argomenti scientifici.

Fig. 3 - Articolo pubblicato sul Corriere del Golfo in occasione della Seconda riunione straordinaria della SISN, La Spezia 1865. Biblioteca MSNM, fondo Cornalia, busta 13, fasc. 6. 
Al termine della riunione di La Spezia, il celebre naturalista toscano Adolfo Targioni Tozzetti (1823-1902), professore di zoologia all'Istituto superiore di Firenze, improvvisò un discorso in cui ribadì la necessità "che alle sommità scientifiche già esistenti in Italia vengano ad aggregarsi molti e molti naturalisti minori, i quali raccolgano fatti e oggetti, per concorrere tutti alla miglior conoscenza del paese, e al progresso della scienza"64. Anch'egli tornò poi sul tema della professionalizzazione del naturalista e della sua missione divulgativa, auspicando "che venga un tempo, nel quale il naturalista non sia più per i profani un essere strano, qualche cosa come una specie particolare del genere uomo, un oggetto di semplice curiosità e di riso per il volgo, ma tutti, grandi e piccoli, dotti e indotti, tutti gli Italiani conoscano a che tendono e che fanno i naturalisti, e vengano loro in soccorso in ogni maniera" 65 .

Anche questa seconda riunione straordinaria fruttò alla SISN l'adesione immediata di 22 nuovi soci effettivi e di due soci corrispondenti.

L'anno 1866 si aprì poi con diversi preparativi, innanzitutto a causa del trasloco della sede della SISN presso il MSNM, finalmente autorizzato dal Comune; in secondo luogo per l'organizzazione del primo Congresso paletnologico a Neuchâtel dal 22 al 25 agosto, in occasione della riunione annuale della Società elvetica di scienze naturali; e in terzo luogo per la preparazione della terza riunione straordinaria fissata ad Ancona, che si sarebbe dovuta tenere dopo il Congresso di Neuchâtel e prima di quello generale degli scienziati italiani in programma a Napoli dal 9 settembre ${ }^{66}$.

Ma ancora una volta le vicende politiche vennero ad arrestare bruscamente i progetti in corso. Se infatti il trasferimento della sede sociale della SISN avvenne effettivamente nel maggio 1866, allo scoppio della terza guerra d'indipendenza, in giugno, ogni attività fu sospesa. La riunione straordinaria di Ancona fu rimandata all'anno successivo e nessun membro della SISN - che pure l'aveva promosso poté recarsi al congresso paletnologico di Neuchâtel ${ }^{67}$.

Il conflitto, come è noto, si concluse in ottobre con la disfatta italiana, il trionfo dell'alleato prussiano e la poco onorevole consegna del Veneto alla Francia e da quest'ultima all'Italia. La liberazione del Veneto dall'Austria, accolta con grande entusiasmo dai patrioti, ebbe ripercussioni immediate anche sulle attività della SISN. Nella seduta del 31 marzo 1867 venne letta ai soci una lettera della Giunta municipale di Vicenza la quale - su suggerimento del naturalista Paolo Lioy (18341911) - si offriva di ospitare il prossimo congresso della Società: "Non appena, dopo le angosciose peripezie della liberazione dallo straniero, le febbrili commozioni dei primi giorni della libertà, rientrava negli agitati animi di questa cittadinanza quella calma che rinnovella le predilette abitudini della vita sociale, sorgeva in Vicenza un desiderio, che da chi conosca le condizioni fisiche della Provincia, cui la città è capoluogo, potrà ben di leggieri comprendersi [...] né il voto dell'egregio naturalista espresso d'invitare la Società italiana di scienze a tenere in Vicenza e provincia il suo Congresso del prossimo settembre poteva venire ad orecchie più desiderose d'accoglierlo con tutto fervore" ${ }^{\prime 68}$.

La risposta positiva del presidente Cornalia non si fece attendere e puntò a rinsaldare il legame che si era già instaurato nei due precedenti congressi di Biella e di La Spezia, dove i naturalisti veneti, ancora sudditi austriaci, avevano incontrato i colleghi ormai "italiani": "Il cortese invito suonava perfettamente d'accordo coi voti tante volte espressi assai prima che spuntasse sulla Venezia l'aurora della liberazione, colle cordiali promesse, coi patti fin d'allora scambiati tra i soci di tutte le 
provincie italiane libere o schiave, cui la tirannide straniera non impedì di abbracciarsi nei geniali congressi di Biella e della Spezia. La riunione dei naturalisti italiani sul veneto suolo sarebbe il primo saluto della scienza alla libertà"69.

In merito alla presidenza del congresso, la SISN accettava la proposta dei vicentini di affidarla al geologo senatore Lodovico Pasini (1804-1870), che pochi mesi dopo, il 26 maggio 1867, entrò a far parte della Società come socio effettivo. Egli tuttavia rifiutò l'incarico e in luglio fu sostituito da Paolo Lioy.

$\mathrm{Ma}$ anche questa volta non fu possibile tenere il congresso. Il colera, la malattia epidemica più temuta dell'Ottocento, tornò a visitare le province italiane ad aprile, partendo dal sud Italia e spostandosi a nord nell'estate. Gli assembramenti di persone apparvero allora come possibili focolai di contagio e gli organizzatori decisero quindi di rimandare la riunione della Società all'anno successivo ${ }^{70}$.

Intanto era giunta alla presidenza della SISN dal socio Edoardo Rostan (18261895), medico e botanico, una prima elaborazione statistica dei soci suddivisi per aree geografiche. La Società prendeva dunque atto per la prima volta della propria diffusione sul territorio italiano: se la stragrande maggioranza dei soci effettivi (324 complessivi) proveniva dalla Lombardia e dal Veneto (196), gli altri erano distribuiti prevalentemente in Piemonte (47) e nella zona della Toscana e delle Marche (55). La città più rappresentata era ovviamente Milano, con i suoi 129 soci, seguita a molta distanza da Firenze e Torino (ciascuna con 15 soci), Pavia (13), Como (10), Genova (9), Bergamo (7), Bologna, Brescia e Spezia (ciascuna con 6 soci), Napoli (5), Ancona, Casale, Livorno, Monza, Perugia, Pisa e Vercelli (tutte con 4 soci) e così via. Vi erano infine 29 soci corrispondenti dall'estero residenti prevalentemente in Francia (8) e in Svizzera (6), e in misura minore in Austria (4) Inghilterra (4) e Prussia (3)

Finalmente nel 1868 fu possibile riprendere l'attività congressuale: la terza riunione straordinaria della SISN si svolse al Teatro Olimpico di Vicenza dal 14 al 17 settembre, sotto la presidenza di Paolo Lioy, che - come previsto dal regolamento - tenne una relazione Sulle condizioni fisiche ed economiche del Vicentino, non mancando tuttavia di ricordare in apertura le vicende politiche che avevano scosso il paese e il filo che legava quel congresso ai precedenti: "E bene mi auguro che riprendansi le riunioni interrotte prima della guerra nazionale, alla quale dobbiamo la indipendenza e la libertà, poi sciaguratamente sospese dai morbi epidemici che serpeggiarono nella Penisola. Ormai questo stringerci le destre, questo comunicarci i nostri studi, questa vita insieme per pochi giorni sì ma che pure basta allo scambio delle idee ed alla alleanza negli utili propositi, questo trasportarci pellegrinando di regione in regione d'Italia non dee passare anno senza che si rinnovi, e così nei modesti convegni noi in breve raggiungeremo lo intento di completamente illustrare nei riguardi naturali la patria nostra, scopo che per ugual via ottennero le società scientifiche tedesche e svizzere, e che la illustre Presidenza della nostra società di scienze si è prefisso quando stabilì le annue riunioni" 72 .

Se da una parte Lioy attribuiva la causa dello scarso progresso scientifico del Vicentino alla soffocante politica austriaca del recente passato ("Siamo un gigante che per anni ed anni ha affaticato a levarsi di dosso la montagna che lo schiacciava; vi è finalmente riuscito, ma gli restano ancora le membra aggranchiate e intorpidite"73), dall'altra vedeva nell'avvenire la possibilità di grandi progressi, che sarebbero passati soprattutto attraverso il potenziamento delle scuole e degli istituti tecnici, la diffusione dei comizi agrari e delle associazioni di credito e di mutuo soccorso, lo sviluppo dei trasporti nazionali e internazionali ${ }^{74}$. 
Al congresso di Vicenza partecipò anche un socio che cominciava in quegli anni a far parlare di sé nell'ambiente medico e naturalistico: Cesare Lombroso (18351909), professore a Pavia di clinica delle malattie mentali e antropologia. Egli presentò la sua memoria Sui caratteri fisici delle diverse popolazioni italiane, in cui ipotizzava "che forse colle differenze fisiche esistenti fra le diverse popolazioni si possono spiegare molte lotte antiche e moderne avvenute fra queste" e concludeva "di aver fede in un migliore avvenire per gl'Italiani, se sapranno saggiamente applicare e utilizzare le loro facoltà fisiche e intellettuali" 75 .

Nella Sezione di zoologia - che ebbe come presidente Cornalia e come segretario Giovanni Canestrini - fu invece presentato il programma di costituzione, a Firenze, di un'associazione "destinata a promuovere e pubblicare un giornale italiano di botanica e di zoologia intitolato Malpighi", promosso dal socio Targioni Tozzetti e finalizzato a riunire in un'unica sede tutte le pubblicazioni naturalistiche che apparivano ancora disperse in troppi e poco conosciuti periodici specialistici ${ }^{76}$. Non sembra tuttavia che il giornale sia stato poi effettivamente pubblicato ${ }^{77}$.

Infine nella seduta generale di chiusura, il 17 settembre 1868, Charles Darwin (1809-1882) fu nominato a pieni voti, con acclamazione, socio corrispondente della SISN ${ }^{78}$.

L'anno 1869, aperto con la seduta del 31 gennaio, vide la Società alle prese con i problemi del bilancio. Il vicesegretario Camillo Marinoni (1845-1882), assistente al MSNM, presentò un rapporto nel quale chiedeva che si costringessero i molti soci morosi al pagamento delle quote arretrate ${ }^{79}$. Le spese per la pubblicazione degli atti del congresso di Vicenza avevano contribuito inoltre ad aggravare la situazione finanziaria, insieme alle "accresciute le spese di porto e di corrispondenza" dovute alla "importanza sempre maggiore che va prendendo la Società, principalmente all'estero", soprattutto in Germania e in America ${ }^{80}$.

A fronte di tutto ciò, la presidenza decise di espellere i soci che non avevano pagato le quote arretrate degli anni 1865-1868 e di tagliare le spese di pubblicazione degli Atti e delle Memorie, riducendo nei primi lo spazio occupato dalle comunicazioni scientifiche e sollecitando i soci ad abbonarsi alle seconde. Nonostante tutto, comunque, il numero dei soci effettivi era cresciuto a 270 , si contavano 33 soci corrispondenti fra i quali si annoveravano i più celebri naturalisti stranieri (tra cui Darwin e l'antropologo Joseph Barnard Davis, eletti l'anno precedente ${ }^{81}$ ), mentre lo scambio delle pubblicazioni avveniva ormai con 80 società e accademie "consorelle negli studj, specialmente all'estero"

Nella seduta del 28 febbraio 1869 si stabilì che, tra le due sedi di Modena e Catania proposte per la successiva riunione straordinaria della SISN, si scegliesse Catania, preferita dalla maggior parte dei soci. Tale scelta tuttavia comportava una maggiore spesa di trasporto, a causa delle difficoltà di raggiungere la Sicilia, ma il ministro dei lavori pubblici, il geologo Lodovico Pasini, assicurò la disponibilità delle compagnie di navigazione Peirano Danovaro, Florio e Rubattino a trasportare gratuitamente i congressisti nell'isola ${ }^{83}$.

La quarta riunione straordinaria della SISN si tenne dunque a Catania dal 23 al 26 agosto 1869 (Fig. 4), sotto la presidenza di Andrea Aradas (1810-1882), professore in quella Università e direttore dell'Accademia Gioenia di scienze naturali. Cornalia, però, qualche giorno prima del congresso inviò una lettera scusandosi di non poter intervenire per "imperiose ragioni" che lo trattenevano in Lombardia ${ }^{84}$. La riunione si aprì quindi senza il rappresentante principale della Società che la promuoveva, sostituito dal segretario Omboni. 


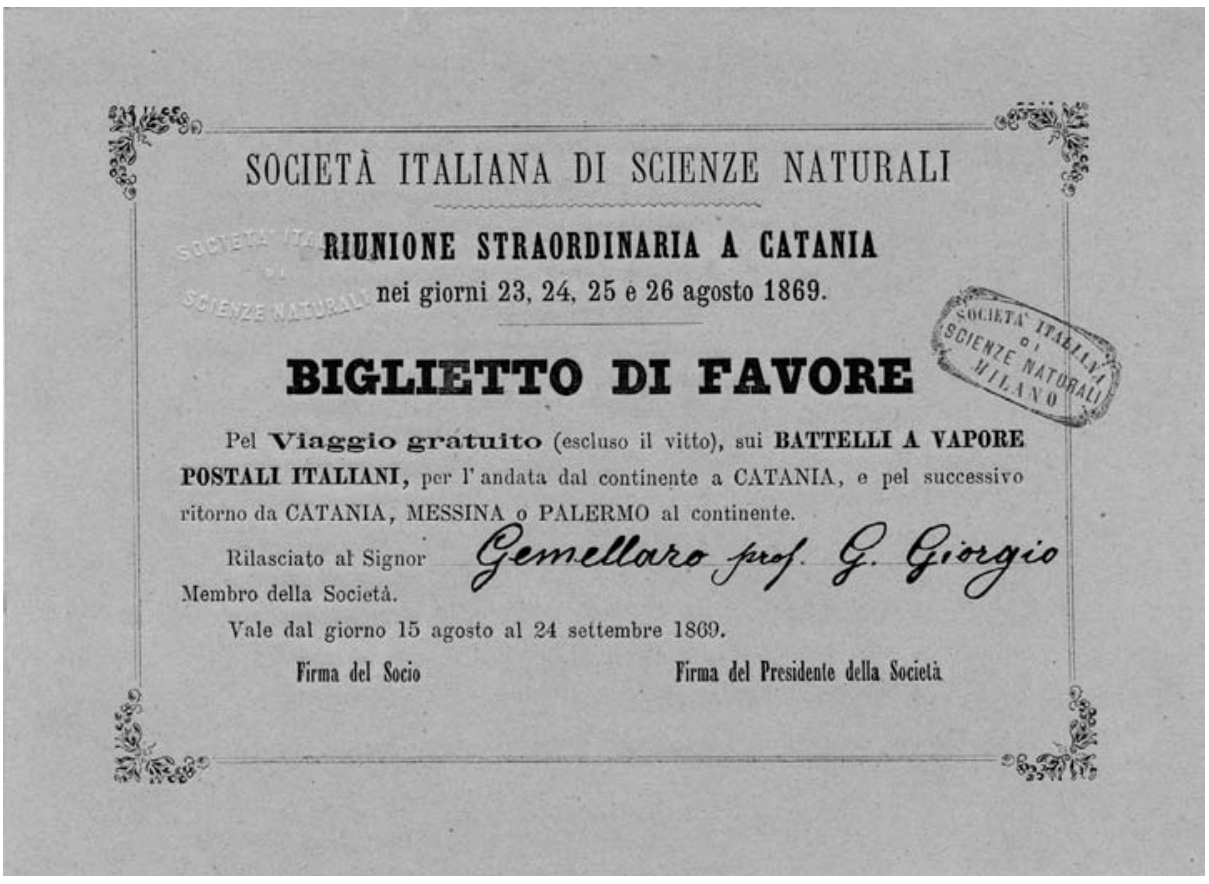

Fig. 4 - Biglietto omaggio di andata e ritorno sui Battelli a vapore postali italiani in favore di soci SISN, in occasione della Quarta riunione straordinaria, Catania 1869. Biblioteca MSNM, fondo SISN, busta 1859-1869, fasc. Congresso Catania, sottofasc. Circolari ed atti relativi della Presidenza ordinaria della Società

Aradas, nel suo discorso inaugurale in cui propose un Abbozzo del panorama etneo, fece voti affinché si stabilisse un collegamento più duraturo tra la SISN e l'Accademia Gioenia, due società accomunate non solo dagli stessi argomenti di studio, ma anche - ancora una volta - dall'appartenenza alla nazionalità italiana ${ }^{85}$.

Il congresso vide molto affollata soprattutto la sezione di zoologia, presieduta da Paolo Panceri, dove Salvatore Trinchese (1836-1897) illustrò gli studi compiuti sugli esemplari di orangutan raccolti da Odoardo Beccari (1843-1920) e da Giacomo Doria durante un viaggio nel Borneo, compiendo un'analisi anatomica comparata rispetto a quelli conservati presso diversi musei italiani e stranieri.

Il congresso si chiuse poi con un'escursione (ad Aci Castello, ai Faraglioni e ad Aci Reale) e con l'ascensione all'Etna da parte di alcuni soci, ansiosi di osservare da vicino le caratteristiche geologiche e naturalistiche del vulcano.

Ma la grande stagione dei congressi postunitari della SISN sembrava ormai in fase declinante. I problemi finanziari della Società; le dimissioni presentate il 28 novembre 1869 dal segretario Omboni, chiamato a insegnare all'Università di Padova e sostituito dal suo vice Camillo Marinoni; il successo sempre più evidente dei congressi di paletnologia organizzati su impulso della Società stessa e forse più interessanti agli occhi di alcuni soci e dello stesso presidente Cornalia, fecero sì che si annullasse il congresso del 1870 che doveva tenersi all'Isola d'Elba. La spiegazione, addotta nella seduta del 27 febbraio 1870, fu la seguente: "La presidenza, avendo incontrate molte difficoltà pratiche alla esecuzione di codesto voto, 
e spinta d'altra parte dal desiderio di rendere più numeroso e brillante il Congresso di paleoetnologia che si riunirà a Bologna, Congresso, che come è noto, è una emanazione della Società italiana di scienze naturali, propone di non tenere per l'anno corrente la riunione straordinaria, nella persuasione che i socj accorreranno a Bologna a prendere parte al Congresso preistorico. I socj Strobel e Bellotti esprimono il desiderio che la presidenza trovi almeno il mezzo di rendere possibile ai membri della Società che formeranno parte del Congresso di Bologna, di riunirsi fra loro e trattare argomenti di zoologia, di botanica e di geologia, in modo da impedire un assoluto e completo assorbimento dei varj rami delle scienze naturali in quello unico della paleoetnologia. La presidenza prende impegno di trattare su queste basi colla presidenza del Congresso preistorico" 86 .

La seduta si concluse quindi con la votazione, a grande maggioranza, di un ordine del giorno in cui, dopo aver ricordato che "scopo precipuo della riunione straordinaria è quello di raccogliere in un'epoca e località determinate il maggior numero possibile di scienziati italiani" "87, si concordava sull'opportunità di dare la precedenza al congresso di paletnologia, che essendo internazionale e trattando comunque argomenti che rientravano "nel campo degli studj di tutte le varie classi di naturalisti", avrebbe esercitato "inevitabilmente una influenza assorbente" 88 , rendendo di fatto superflue, per quell'anno, altre riunioni.

\section{L'inizio del declino}

Furono ancora una volta le vicende politiche, però, a influenzare il calendario delle attività della SISN. La questione romana, diventata incandescente nell'estate del 1870 e conclusasi il 20 settembre con la presa di Roma e il crollo dello Stato Pontificio, rese di fatto impossibile organizzare il quinto Congresso paletnologico, che fu rimandato all'ottobre dell'anno successivo, sempre a Bologna, sotto la presidenza del senatore conte Giovanni Gozzadini. Si decise allora che anche la quinta riunione straordinaria della SISN si sarebbe tenuta contemporaneamente al congresso, ma "in forma privata, allo scopo sia di comunicazioni scientifiche che di fissare il congresso per il 1872" $"$. Nella circolare di invito ai soci si leggeva: "Occasione più propizia di questa, in cui i più dotti naturalisti d'ogni parte d'Europa si danno convegno per discutere sulle prime origini dell'uomo e per far note le indagini fatte, le proprie idee e le nuove importantissime scoperte, non si potrebbe offrire altra volta ai naturalisti italiani, e a tutti coloro che seguono con interesse lo sviluppo dell'ingegno umano" .

Se la quinta riunione straordinaria della SISN fu dunque in realtà solo un convegno privato tenutosi il 6 ottobre 1871 in una sala dell'Archiginnasio ${ }^{91}$, il quinto Congresso internazionale di antropologia e di archeologia preistorica fu invece un successo, coronato dalla presenza del principe Umberto e del ministro della pubblica istruzione Cesare Correnti; il presidente Gozzadini fece un riassunto degli studi preistorici effettuati in Italia nell'ultimo decennio, ovvero da quando era nata in Italia l'archeologia preistorica, mentre il MSNM partecipò all'Esposizione preistorica esponendo la raccolta delle palafitte di Varese donatagli dalla SISN ${ }^{92}$.

Tra la fine del 1871 e l'inizio del 1872 i soci scelsero Siena come sede della sesta riunione straordinaria della Società, preferendola a Pisa, mentre alla presidenza fu nominato Giovanni Campani, docente di chimica nell'Università senese. Il congresso fu fissato dal 22 al 25 settembre 1872 e la segreteria affidata a Camillo Marinoni. Ancora una volta, tuttavia, Cornalia si scusò di non poter intervenire e 
toccò a Marinoni fare le sue veci. Egli tenne una relazione Sullo stato morale e materiale della Società italiana di scienze naturali nell'anno $1872^{93}$, in cui riprese sostanzialmente i concetti esposti dallo stesso Cornalia al primo congresso di Biella del 1864 e ribadì il ruolo fondamentale della scienza e dei congressi nel processo di costruzione della nazione ${ }^{94}$. Secondo Marinoni, le riunioni straordinarie avevano dato un evidente impulso all'attività scientifica della Società, tanto che a Biella, nel 1864, era stata deciso di dare inizio alla pubblicazione delle Memorie - "la più bella e ricca pubblicazione del genere che si faccia fino ad ora in Italia" - che venne ad affiancarsi a quella già in corso degli Atti. In questo modo molti studiosi, "anche estranei alla Società, affidarono ad essa il loro nome e l'avvenire dei loro studii" 95 , aggiungendo i propri articoli a quelli del gruppo originario prevalentemente milanese e lombardo. Tra i nuovi autori, Marinoni segnalava Aradas, Ascherson, Claparède, Cocchi, D’Achiardi, Garovaglio, Gibelli, Issel, Lioy, Scarabelli, Seguenza, Steinheil e Targioni Tozzetti, ma non poteva non evidenziare il problema finanziario che la pubblicazione delle Memorie continuava a porre alla Società e invitava quindi i soci a contribuire e sostenerne le spese.

Ricordava poi il ruolo della SISN nella promozione di esplorazioni scientifiche e ricerche preistoriche, soprattutto in Lombardia, che avevano consentito al MSNM di acquisire "la più gran parte di quei monumenti dell'industria dei nostri avi che si ammirano oggi da tutti" $"$.

Infine ribadiva l'importanza delle riunioni straordinarie come trampolini di lancio e casse di risonanza per nuove iniziative: a La Spezia, ad esempio, era stato fondato il Congresso internazionale di antropologia e di archeologia preistorica, "il quale, già fatto gigante, gira le capitali del mondo"; dal congresso di Vicenza erano stati indirizzati al governo "lagni e proposte sulla legge della caccia, a fine di porre un argine all'invasione di tante specie d'insetti dannosi all'agricoltura"; mentre a Catania si era discusso "sulla malattia degli agrumi in Sicilia"97.

Anche il congresso di Siena ebbe successo: vi parteciparono circa ottanta persone e tutti gli istituti scientifici e i principali stabilimenti della città rimasero aperti per i congressisti. La Società entomologica italiana, presieduta da Adolfo Targioni Tozzetti, tenne inoltre negli stessi giorni la sua seconda adunanza generale, in modo che i soci potessero partecipare al congresso, nel quale fu aggiunta infatti una quarta sezione di entomologia.

Tuttavia, come si è detto, la grande stagione delle sedute straordinarie della SISN aveva cominciato il suo lento declino. Dagli Atti traspare una certa stanchezza nel rinnovarne la tradizione e una crescente difficoltà a trovare persone disponibili ad accollarsi l'onere della presidenza, che comportava un impegno organizzativo non indifferente in loco. Inoltre, se già da tempo si sentiva la mancanza di Omboni (ormai definitivamente a Padova) come segretario, occorreva ora nominarne un altro al posto di Marinoni, chiamato a insegnare presso il Regio Istituto tecnico provinciale di Caserta (di lì a poco sarebbe stato sostituito da Ferdinando Sordelli, aggiunto al MSNM). Vista la quantità di lavoro che continuava ad accumularsi sul tavolo del segretario, poi, fu necessario nominare un assistente, Ettore Colombo, per le mansioni ordinarie di spedizione degli Atti e delle circolari, di tenuta dei registri e di disbrigo della corrispondenza con i soci, compensandolo con una "piccola retribuzione" 98 .

Nel frattempo, la scelta della sede per la riunione straordinaria del 1873 era caduta su Sassari, anche se da tempo Omboni aveva fatto notare che l'Esposizione universale prevista a Vienna avrebbe ostacolato la presenza di molti soci. Questi, 
infatti, nella seduta del 7 luglio 1873, confermarono l'opportunità di rimandare il congresso, preoccupati anche per la nuova epidemia di colera, che in effetti insieme al disastro finanziario seguito al crollo improvviso della borsa - fece poi fuggire i visitatori anche dall'Esposizione viennese. Inoltre si rilevò che "il breve intervallo di un anno fra l'una e l'altra riunione straordinaria" lasciava poco tempo per effettuare quei "lunghi e meditati studj" necessari a produrre "molte ed importanti comunicazioni scientifiche" e quindi "venendo ad essere limitato il campo della discussione, minore sentesi il bisogno di personali ritrovi per gli studiosi" ${ }^{99}$.

Vi era poi una sorta di vuoto direttivo dovuto all'assenza di Cornalia dagli ultimi due congressi, seguita dall'annuncio, il 28 dicembre 1873, della sua prossima partenza per un viaggio scientifico in Egitto, effettuato nella prima metà del 1874 . Del resto il ritratto che di Cornalia fece Stoppani dopo la sua morte ci restituisce l'immagine di un uomo cagionevole di salute, "cercato, trascinato sbattuto da tutte le parti, e costretto ad assumere ogni giorno nuovi impegni ed a rispondere a nuovi quesiti", ma anche per questo figlio dei tempi, di "quel movimento convulso che agita in oggi la società in tutti i sensi [e] crea tali esigenze e tali occasioni che, per chi ha appena un po' di mente e un po' di volontà, il tempo di studiare e di scrivere diventa merce di furto" 100 .

Senza Cornalia, senza Omboni, alle prese con una crescente burocrazia da gestire e con un'evidente disaffezione da parte dei soci, la SISN cominciava dunque nei primi anni settanta dell'Ottocento a dare segni di decadenza. Sospesa la pubblicazione delle Memorie a causa delle difficoltà economiche, si decise nel febbraio 1874 di modificare anche l'articolo del regolamento relativo ai soci corrispondenti, introducendo per loro l'obbligo di operare per il bene e lo sviluppo della società (trovando contatti, procurando pubblicazioni e così via), pena l'essere depennati dopo tre anni dall'elenco dei soci stessi. Inoltre la crisi generale della SISN era aggravata dalla concorrenza di numerose altre società scientifiche sempre più specialistiche, nate nel frattempo su tutto il territorio italiano, che contribuivano a toglierle soci e spazi di intervento.

In questo quadro si comprende perché il 28 giugno 1874 Cornalia, rientrato dal suo viaggio, proponesse ai soci di accettare l'offerta di Omboni, ora segretario della Società veneto-trentina di scienze a Padova, di partecipare al Congresso dei naturalisti italiani organizzato da quella Società ad Arco, in Trentino, nell'autunno successivo ${ }^{101}$.

La partecipazione alla riunione della SISN tenutasi nell'ambito del Congresso di Arco del 21-24 settembre, presieduto da Prospero Marchetti (1822-1884) con la segreteria di Omboni, fu tuttavia molto scarsa, nonostante fossero state invitate, oltre alla SISN, la Società entomologica, la Società dei naturalisti di Modena, l'Associazione dei medici e naturalisti di Napoli, la Società malacologica di Pisa, la Società agraria di Roveredo, l'Ateneo di Venezia e il Club alpino italiano; si presentarono infatti solo 27 persone, di cui 6 appartenenti alla SISN e il convegno non fu dunque annoverato neppure tra le riunioni straordinarie vere e proprie, passando quasi inosservato ${ }^{102}$.

Poco dopo, il 15 novembre 1874, moriva a Milano Giuseppe Balsamo Crivelli, vero punto di riferimento della generazione risorgimentale dei naturalisti italiani e maestro di molti degli studiosi che in quegli stessi anni occupavano le cattedre universitarie e popolavano gli istituti e i musei naturalistici del Paese. La stanchezza della SISN era in effetti molto legata anche all'età ormai avanzata dei suoi soci fondatori. 
L'ultima riunione straordinaria del periodo postunitario, la settima, si tenne a Varese dal 24 al 27 settembre 1878, "nel magnifico salone del palazzo Veratti"103. La presiedette Leopoldo Maggi (1840-1905), professore di zoologia e anatomia comparata e direttore del Museo di Storia Naturale dell’Università di Pavia. Nel suo discorso d'apertura Intorno alle condizioni naturali del territorio varesino, ormai l'accento patriottico dei primi congressi trovava eco solo in un breve accenno a Varese come "degna cooperatrice all'umana civiltà, e vera figlia dell'Italia", una città che non aveva mancato di pagare "il suo tributo di sangue in tutte le patrie battaglie"104. Maggi ricordò inoltre l'istituzione, nel 1871, del Museo patrio della città, dove era stata allestita una sezione storico-archeologica e di paleontologia, che i congressisti poterono visitare.

Questa volta Cornalia fu presente al congresso, insieme al segretario Sordelli, e assunse la presidenza della sezione di "zoologia, anatomia comparata e paletnologia dei vertebrati". Le altre tre sezioni, rispettivamente presiedute da Giuseppe Gibelli (dell'Università di Modena), Giovanni Omboni e Ippolito Martelli-Bolognini di Pistoia, erano quelle di "botanica e agricoltura", "geologia, mineralogia e chimica" e "paletnologia".

Una delle novità introdotte nella sezione di zoologia fu la discussione sulle misure da prendere contro la possibile invasione della fillossera, il parassita che da qualche anno stava devastando la viticoltura europea e che di lì a poco, nel 1879, avrebbe effettivamente raggiunto l'Italia. Il socio Felice Franceschini propose che la SISN organizzasse "un indeterminato numero di vedette antifillosseriche, ciascuna delle quali tenga in osservazione una data zona, informi la Presidenza nello sgraziato caso che la fillossera comparisse fra noi, onde la Società reclami quegli energici provvedimenti governativi che si crederanno opportuni"105. La proposta fu votata all'unanimità nella seduta conclusiva del congresso.

La settima riunione straordinaria di Varese, contrariamente alle precedenti, non si chiuse con uno sguardo al futuro, ovvero con la consueta scelta della sede del congresso successivo (nonostante la proposta di Marinoni di tenerlo a Udine), bensì con la decisione di Cornalia di procrastinare la decisione, dal momento che l'intervallo di un solo anno tra un congresso e l'altro gli sembrava troppo breve ${ }^{106}$.

Gli anni settanta dell'Ottocento avevano del resto segnato da un lato l'apice del successo della SISN, che proprio nel 1870 aveva raggiunto il suo numero massimo di iscritti (301), dall'altro l'inizio della sua decadenza. L'avvenuta unificazione del paese, il grande sogno dei soci fondatori, aveva tolto all'impresa dei congressi itineranti gran parte del suo fascino e della sua spinta ideale verso la costituzione di una comunità "italiana" di naturalisti, che riunisse "i molti studiosi sparsi e divisi in tanti grandi e piccoli centri" i quali proprio grazie alla SISN "poterono conoscersi, avvicinarsi, intendersi"107. Questo ruolo era stato tutto sommato svolto bene dalla Società, ma si stava esaurendo, come quello generale dei congressi degli scienziati risorgimentali, che ormai lasciava il posto alle esigenze dello Stato di centralizzare a Roma l'eccellenza "ufficiale" e di consolidare in questo contesto la funzione dell'università e dell' Accademia dei Lincei ${ }^{108}$.

Alla morte di Cornalia nel 1882, la direzione del MSNM e la presidenza della SISN passarono ad Antonio Stoppani, il quale aprì la seduta del 29 aprile 1883 invitando i soci a nominare una commissione che studiasse "il modo di dar nuovo impulso alla vita della nostra società". Era dunque ormai sotto gli occhi di tutti che la SISN navigava in cattive acque. La discussione fu vivace e portò all'attenzione generale le cause esterne ed interne della crisi, riassumibili sostanzialmente da un 
lato nella concorrenza delle nuove associazioni naturalistiche di carattere locale e specialistico, dall'altro in un rallentamento generale della produzione scientifica della Società, evidente con la sospensione della pubblicazione dalle Memorie e la mancata organizzazione di nuove riunioni straordinarie fuori sede (le due cose erano strettamente correlate tra loro, come ricordava nel 1885 il segretario Pini: "Per produzione di lavori, le annate più feconde furono generalmente quelle in cui la Società tenne le sue adunanze straordinarie"109).

Per quanto riguardava il primo punto, il socio Pietro Pavesi riteneva necessario "radunare le forze disperse" e ricostituire la società su nuove basi, "in modo che essa possa farsi centro ove mettano capo le altre, a guisa della Società Elvetica, che racchiude le società di Berna, Ginevra, Neuchâtel, ecc."110. Ancora una volta dunque, il modello di riferimento era la Società elvetica di scienze naturali e la sua organizzazione federalistica. A Pavesi facevano poi eco Francesco Salmoiraghi e Torquato Taramelli, il primo portando un altro esempio di associazione che riuniva diverse anime, quello del Club alpino italiano, il secondo ricordando l'utilità che sarebbe derivata "alla scienza ed a tutti i soci d'ogni singola società", se le diverse associazioni naturalistiche si fossero "riannodate" alla SISN, la "loro madre naturale", "la prima sorta in Italia e quella che anche oggidì conta maggior numero di soci" "111.

Del resto, come ricordava Pini, "il progredire incessante di alcuni rami speciali delle scienze" aveva accelerato il processo di costituzione di molte associazioni specialistiche in tutta Italia ${ }^{112}$. Quanto alla necessità di ripristinare i congressi espressa dal socio Cristoforo Bellotti, poi, Pini obbiettava che "le finanze sociali compromesse col congresso di Varese, benché migliorate, non [erano] ancora in istato da permettere spese straordinarie come quelle inseparabili da un congresso" 113 .

Il problema pressante del reperimento delle risorse economiche si rese ancor più manifesto nelle sedute successive del 3 giugno e del $1^{\circ}$ luglio 1883 , quando Pavesi e Taramelli presentarono un pacchetto di proposte di riforma della Società sostanzialmente finalizzate a questo scopo, alcune delle quali furono approvate e inserite nel regolamento: si istituiva una classe di soci effettivi studenti ai quali era richiesta una quota associativa inferiore; si stabiliva una diminuzione delle quote in generale a partire dal momento in cui la cifra complessiva dei soci avesse raggiunto il numero di 300; si aboliva la categoria dei soci corrispondenti; si aprivano le sedute della Società al pubblico, dandone notizia nei principali giornali cittadini; si stabiliva di tenere "invariabilmente tutti gli anni un Congresso in autunno [...], possibilmente fuori Milano", ma in caso di impossibilità anche "in Milano sotto la Presidenza ordinaria, [...] colle norme già stabilite pei congressi scientifici" 114 .

Nonostante queste modifiche, tuttavia, la SISN non organizzò più congressi fino al 1906. Gli anni ottanta si trascinarono stancamente sotto la presidenza di Stoppani, che morì il $1^{\circ}$ gennaio 1891. Le sedute, anziché aumentare, si diradarono sempre di più e i soci, che nel 1870 erano arrivati a essere 301, nel 1884 erano già calati a 141. Il segretario Sordelli aveva dato le dimissioni e nel 1883 era stato sostituito dal geologo Giuseppe Mercalli, affiancato l'anno dopo da Napoleone Pini.

Contemporaneamente, anche il MSNM stava vivendo momenti di difficoltà: entrato nel 1875 a far parte del Consorzio degli istituti d'istruzione superiore, aveva perso gran parte della sua autonomia con il passaggio delle funzioni di indirizzo e di vigilanza (prima riservate al suo Collegio dei conservatori), al Consiglio direttivo del Consorzio stesso, guidato dal Politecnico e dal suo direttore Francesco Brioschi. Con l'aumento delle collezioni e dell'attività didattica, inoltre, era divenuto 
sempre più urgente trovare nuovi spazi e a partire dal 1884 si cominciò a vagliare l'opportunità di traslocare in una sede più spaziosa. Questo progetto si realizzò tra il 1888 e il 1890, con la costruzione del nuovo edificio in via Palestro, dove il Museo si trasferì definitivamente nel 1893, in concomitanza con il vuoto direttivo causato dalla morte di Stoppani.

Anche gli Atti della SISN rispecchiano il momento di crisi: il volume del 1890 è infatti molto ridotto, quello del 1891 non fu mai pubblicato e quelli degli anni 1892-1894 furono pubblicati insieme in un volume cumulativo.

\section{Una nuova epoca}

Quella che si aprì nel 1892 fu una nuova fase nella storia della Società, che sotto la presidenza del geologo Gaetano Negri (1838-1921) dal 1892 al 1894 e poi dell'astronomo Giovanni Celoria (1842-1920) dal 1895 al 1899 conobbe un nuovo sviluppo, aprendo le porte a una nuova classe dirigente e a una nuova generazione di studiosi, non più e non tanto animati da una visione patriottica e "unificatrice" della scienza, quanto piuttosto da una nuova fiducia nella scienza stessa - ormai sempre più specialistica - in quanto motore dello sviluppo economico e industriale. Negli anni novanta, dunque, la parola d'ordine tornò ad essere "Milano", la Milano in fase di decollo industriale e in cui, grazie al già citato Consorzio, si stava realizzando una grande rete di istituti d'alta cultura, all'interno della quale il MSNM aveva un ruolo fondamentale come polo degli studi biologici115.

Anche il legame tra il Museo civico e la SISN allora cambiò, in quanto da un lato con la morte di Stoppani cessò la consuetudine di avere come presidente della Società il direttore del Museo, dall'altro la collaborazione si fece più stretta ma più paritaria, tanto che nel 1896 grazie al contributo del Comune (di cui Celoria era assessore) si diede inizio alla pubblicazione congiunta degli Atti della Società Italiana di Scienze Naturali e del Museo Civico di Storia Naturale in Milano. Il connubio risultò favorevole e nel giro di pochissimi anni il numero dei soci aumentò visibilmente, con l'entrata nella SISN di quasi tutti i direttori e gli studiosi degli istituti milanesi consorziati al Politecnico.

Per ovviare ai problemi economici della Società, invece, nel nuovo Regolamento del 1895 fu introdotta la figura dei soci benemeriti, ovvero di coloro che "mediante cospicue elargizioni" contribuivano a consolidare il capitale sociale ${ }^{116}$. Il primo socio benemerito fu, quello stesso anno, Cristoforo Bellotti, che donò alla SISN la somma di 5000 lire su due libretti della Cassa di risparmio di Varese ${ }^{117}$. Al posto delle "riunioni straordinarie", inoltre, si stabilì che la Società avrebbe potuto indire dei più moderni "congressi scientifici", stabilendone di volta in volta la sede e le norme organizzative ${ }^{118}$.

Sia Negri che, soprattutto, Celoria concentrarono l'attenzione sulla realtà milanese, allora in fase di decollo industriale, puntando a "curare l'aumento dei soci residenti", meglio se facoltosi, "acciocché concorrano col loro annuo contributo a rendere sempre maggiore il numero e l'importanza delle pubblicazioni della Società" "119. Tra le cause principali della crisi della SISN, infatti, Celoria annoverava la passata trascuratezza nei confronti degli "elementi locali", che in quanto "fondamenti di stabilità sociale", avrebbero dato "maggior forza di vita" alla Società stessa, come accadeva "in tutte le parti di Europa ove stava risorgendo la scienza" ${ }^{20}$. Si era creduto a lungo, secondo Celoria, che la Società potesse tenersi 
in vita solo con le sue pubblicazioni, ma sarebbe stato molto più utile "istituire delle frequenti sedute speciali destinate precipuamente alle conversazioni scientifiche, le quali, mentre potrebbero offrire molta utilità diretta per la coltura generale di tutti coloro che vi intervenissero e potrebbero preparare il terreno a nuovi ed importanti studi, recherebbero al tempo stesso il grande vantaggio di avvicinare tra loro buon numero di persone colte, valenti e facoltose, servendo come centro di attrazione" $" 121$.

La SISN doveva diventare dunque "un centro di discussione" delle novità scientifiche e "un centro di diffusione" delle stesse ${ }^{122}$. Con le conferenze divulgative sarebbe stato più agevole coinvolgere la borghesia industriale in ascesa, che a Milano non faceva mai mancare il suo appoggio economico alle iniziative votate al bene pubblico, si trattasse di opere benefiche e assistenziali, di studi scientifici o di eventi culturali in genere.

Nel giro di pochi mesi, in un crescendo di successo e d'interesse da parte del pubblico, sotto la guida energica di Celoria la SISN acquisì nel 1896 più di cinquanta nuovi soci, appartenenti per la maggior parte al mondo delle professioni (soprattutto medici e ingegneri). Migliorate progressivamente le condizioni della Società e avviata con successo la stagione delle conferenze, Celoria cercò poi nel 1897 di inserire la SISN in una più vasta rete di associazioni scientifiche, facendola confluire nel progetto allora in corso di Federazione delle associazioni scientifiche milanesi. Ma in questo caso la proposta cadde nel vuoto, soprattutto per il timore di alcuni soci di compromettere il legame con il MSNM, ancora in corso di allestimento nella nuova sede.

Nominato vicepresidente nel maggio 1897 poiché non poteva più essere rinnovato come presidente, Celoria continuò allora sulla strada già avviata del coinvolgimento del maggior numero possibile di "persone facoltose" della città, portando come esempio la creazione dell'Università di Chicago, inaugurata nel 1895, "per la quale quel popolo di commercianti in meno di quattro anni riuscì a mettere insieme la somma di 60 milioni di lire" ${ }^{123}$. Le finalità erano dunque cambiate rispetto al passato: non più il coinvolgimento dei naturalisti della nuova Italia unita, ma quello dei ricchi mecenati interessati alle applicazioni della scienza. Non più un ideale patriottico e nazionale, ma una finalità pratica e legata all'ambito locale. Non più la scienza volta all'affratellamento dei popoli, ma come motore del progresso industriale e commerciale dell'area lombarda.

Comunque sia, le migliorate condizioni della Società tornarono indubbiamente anche a vantaggio del MSNM e sul finire del 1898 lo stesso Celoria si augurava "che le riunioni dei soci porgano sempre più frequenti occasioni ai professori del Museo stesso per far rendere di pubblico interesse tutti gli studi che in questo si fanno" $" 124$.

Nel 1899 scadeva però il suo mandato di vicepresidente (facente funzioni di presidente) e non fu facile trovargli un degno sostituto. Dopo aver tentato di coinvolgere il conte Giberto Borromeo jr. e poi il professor Italo Chelussi, a dicembre i soci elessero presidente l'anziano medico ostetrico Edoardo Porro, coadiuvato dall'ingegnere Francesco Salmojraghi, già vicepresidente da giugno ${ }^{125}$.

La salute malferma e l'età avanzata, tuttavia, non consentirono a Porro di esercitare il suo ruolo con la dovuta serenità e intraprendenza. La proposta presentata da Arturo Issel di organizzare una riunione delle società naturalistiche in occasione del Congresso geografico di Milano del 1901 fu scartata con scuse abbastanza pretestuose $^{126}$, finché il 19 gennaio 1902, Porro - che morì pochi mesi 
dopo - fu sostituito alla presidenza da Cristoforo Bellotti, a sua volta sostituito nel gennaio 1903 da Ettore Artini (1866-1928), direttore della Sezione di mineralogia del MSNM.

Fu tuttavia necessario aspettare il 21 febbraio 1904 perché si tornasse a parlare dei congressi, quando Pompeo Castelfranco, da poco eletto archivista della SISN, aprì una discussione circa "il miglior modo di solennizzare il $50^{\circ}$ anniversario della fondazione della Società e l'opportunità di promuovere pubbliche conferenze scientifiche in seno alla Società stessa", mostrandosi "favorevole all'idea di convocare pel 1906 un congresso di naturalisti" 127.

Anomalo e diverso rispetto ai precedenti, il Congresso dei naturalisti italiani che si tenne a Milano dal 15 al 19 settembre 1906 fu l'ultimo organizzato dalla SISN e l'unico a produrre un volume di atti autonomo ${ }^{128}$. Figlio del nuovo secolo, legato strettamente ai festeggiamenti per il cinquantesimo anniversario della Società (la cui prima seduta risaliva all's febbraio 1856) e al grande evento dell'Esposizione internazionale del Sempione, esso non aveva in realtà più molto a che vedere - anche nella denominazione - con le vecchie "riunioni straordinarie" ottocentesche. Nella circolare d'invito diffusa nel dicembre 1905 si faceva espressamente riferimento alla "milanesità" dell'iniziativa, tanto che i naturalisti erano chiamati a raccolta "affinché la solenne riunione sia d'incremento alla Scienza e di lustro alla Patria nostra, mentre questa s'accinge in Milano a dar prova di operoso progresso e di pacifica espansione, inaugurando nuove vie di comunicazione tra i popoli""129.

Nel discorso di apertura il presidente Artini riprese in parte la tradizione patriottica delle riunioni straordinarie del recente passato, rivolgendosi agli italiani ancora soggetti al dominio straniero: "Vorrei finalmente trovare una parola adatta ad esprimere tutto il vivo compiacimento che proviamo nel veder qui tra noi i colleghi Ticinesi, Trentini e Triestini, i quali accolsero benignamente il nostro invito, rivolto, senza tener conto dei confini di Stato, a tutti coloro che per comunanza di origini e di eloquio posson dirsi fratelli" ${ }^{130}$. Ma poi entrava nel merito del congresso, spiegando lucidamente quali erano le nuove necessità: "In un'epoca nella quale, per la prevalenza della ricerca analitica e la divisione del lavoro spinta all'estremo, il campo di studio è andato frazionandosi sempre più, ben vengano le riunioni generali, che ci permettano di conoscersi e di affiatarci, coordinando le varie ricerche a fini più complessi e più alti"131.

Anche a questo proposito, Artini sosteneva la necessità di ricostituire la Società italiana per il progresso delle scienze (SIPS), nata nel 1862 per rendere stabili e regolari i congressi risorgimentali degli scienziati italiani, la quale dopo un periodo di inattività che durava la 1875 , rinacque effettivamente in quell'occasione ${ }^{132}$. Forse fu anche per questo che la SISN non tenne più congressi dopo il 1906, dal momento che le discipline naturalistiche rientrarono nelle varie classi in cui si articolava l'attività della SIPS.

Ma ormai i tempi erano davvero mutati e se Milano aveva cambiato aspetto "tanto da apparire una città nuova" a chi la rivedesse a pochi anni di distanza, le stesse discipline scientifiche risultavano ora "ampliate" e "trasformate": se infatti alcune teorie si potevano "ritenere del tutto tramontate", altre, fino a qualche anno prima "luminose ed affascinanti", volgevano già "al crepuscolo", mentre si aprivano "nuove ed ampie vie di ricerca [...] sempre più illuminate e sicure, convergenti a quelle scienze fondamentali, che interpretano e misurano le energie operanti della natura" 133 . 
Con il congresso del 1906 si chiudeva dunque un'epoca e se ne apriva una nuova: l'evento era "riuscito un vero plebiscito di stima e simpatia delle società consorelle e degli studiosi" e "data l'apatia che regna[va] nel campo delle scienze naturali", la presenza di 309 congressisti appariva agli organizzatori come "un vero trionfo" $"$ "134.

\section{Conclusione}

Ripercorrendo la storia della SISN, emerge dunque l'immagine di una società decisamente impegnata sul fronte politico, in un periodo storico in cui era del resto difficile separare - almeno in Italia - questo aspetto da quello più strettamente scientifico. Attraverso i congressi, la SISN contribuì a rinsaldare i legami tra i naturalisti italiani, rafforzandone l'immagine di veri scienziati e professionisti, accomunati dall'appartenenza al giovane Stato nazionale, nel suo duplice aspetto di "patria" e di "oggetto di studio", ovvero di territorio da esplorare, studiare e descrivere. Con il compimento dell'Unità, la nascita di nuove associazioni specialistiche e la progressiva scomparsa dei soci fondatori che avevano vissuto direttamente il Risorgimento, la spinta politica venne meno, cessò l'attività congressuale ed ebbe inizio un periodo di ripensamento e di riorganizzazione della Società, la quale si affacciò al nuovo secolo prendendo atto dei profondi mutamenti ormai intervenuti non solo al suo interno, ma nella città che l'aveva vista nascere, nel paese che si avviava alla modernizzazione e nella comunità scientifica sempre più sopraffatta dallo sviluppo accelerato delle scoperte e dei nuovi campi di ricerca.

\section{Note}

\section{Abbreviazioni}

Atti SGM - Atti della Società Geologica residente in Milano. Bernardoni, Milano, 1855-1859.

Atti SISN - Atti della Società Italiana di Scienze Naturali. Bernardoni, Milano, 1860-1895.

Atti SISN-MSNM - Atti della Società Italiana di Scienze Naturali e del Museo Civico di Storia Naturale in Milano. Bernardoni, Milano, 1896-1902; Tipografia degli operai, Milano, 1903-1906.

1 Wilhelm von Haidinger, mineralogista austriaco, primo direttore del Geologische Reichsanstalt, l'I.R. Istituto geologico di Vienna, già direttore del Gabinetto mineralogico della Corte e del locale Museo Montanistico fondato nel 1844. Cfr. Vaccari E., 1999.

2 Si trattava del Museo Reale di Storia Naturale istituito a Milano nel 1809 dal Consiglio delle miniere del Regno italico e un tempo diretto da Giovanni Battista Brocchi (1772-1824). Meglio conosciuto con il nome di Gabinetto di minerali e fossili di Santa Teresa (era infatti situato sullo stradone di Santa Teresa, oggi via Moscova), conservava soprattutto la preziosa collezione paleontologica di Giuseppe Cortesi (1760-1838), costituita da scheletri di cetacei e di conchiglie del Terziario dei terreni argillosi dell'Appennino piacentino.

3 Cfr. Archivio MSNM, Archivio amministrativo, b. 11, fasc. 11/45, lettera di Haidinger a Jan, 27 maggio 1852. Nell'oggetto del fascicolo si legge: «L'I.R. Istituto Nazionale Geologico di Vienna, riferendosi alla propria nota del 20 maggio 1850 e all'opinione espressa dal prof. Jan che qualora la raccolta Cortesi dovesse essere dall'I.R. Governo ceduta alla Città di Milano, l'erigenda Società Geologica non solo ne potrebbe approfittare, ma per essa potrebbe essere anche predisposto qualche locale nel Civico Museo di Milano, sollecita nuovamente il direttore del Museo a voler promuovere l'istituzione della Società Geologica, ora che, come gli risulta da un comunicato del Ministero, la detta raccolta Cortesi venne in data 7 giugno 1851 messa a disposizione della Città di Milano». In realtà la raccolta era stata appunto «messa a disposizione», non effettivamente ceduta al Comune.

4 Archivio SISN, b. 1855-1859, lettera di Ambrogio Robiati a Wilhelm Haidinger, Milano, 18 febbraio 1855.

5 Zocchi P., 2010a. 
6 Regolamento 1858: 30, art. 39.

7 Cfr. l'elenco dei soci fondatori in Atti SGM, 1 (1855-1859): 15-21.

8 Cornalia E., 1859a: 72

$9 \quad$ Ibid.: $72-73$.

10 Ibid: 73

$11 \quad$ Ibid.: 74 .

12 Ibid.: 76

13 Cfr. Cornalia E., 1859b: 547.

14 Lettera di Sella al ministro dell'istruzione pubblica Gabrio Casati, Torino, 26 ottobre 1859. In Quazza G., Quazza M. (a cura di), 1980: 241.

15 Atti SISN, 2 (1859-1860), seduta 18 dicembre 1859: 26.

16 Archivio SISN, b. 1859-1869, fasc. Carteggio 1859-1865, lettera di Oronzio Gabriele Costa a Cornalia, Napoli, 12 febbraio 1859.

17 Atti SISN, 2 (1859-1860), seduta 22 gennaio 1860: 36.

18 Pini N., 1885: 17. Com'è noto, il 6 febbraio 1853, l'ultima domenica di Carnevale, scoppiò a Milano un'insurrezione popolare antiaustriaca di stampo mazziniano. Un migliaio circa di artigiani e operai assaltò all'arma bianca le caserme e i depositi di munizioni degli austriaci, innalzando barricate e aggredendo gli ufficiali in libera uscita. La mancanza di coordinamento, tuttavia, non consentì agli insorti di agire con efficacia e di coinvolgere in massa la popolazione, com'era accaduto nel Quarantotto. Gli austriaci riuscirono quindi a reprimere facilmente la rivolta e ad attuare una feroce repressione, che portò all'arresto di quasi 900 persone e all'esecuzione di 16 condanne a morte.

19 Atti SISN, 2 (1860), seduta 22 aprile 1860: 113.

20 Cornalia E., 1860: 200-202.

21 Omboni G., 1860: 313.

22 Ibid.

23 Ibid: 314.

24 Cfr. Casalena M.P., 2007.

25 Regolamento 1858: 27, art. 23-24.

26 Atti SISN, 3 (1861), seduta 28 luglio 1861: 355.

27 Atti SISN, 3 (1861), seduta 24 novembre 1861: 404.

28 Biblioteca MSNM, Fondo Cornalia, b. 13, fasc. 6, lettera di Pillet a Cornalia, Chambéry, 3 marzo 1863.

29 Ibid., circolare a stampa firmata dal presidente Cornalia e dal segretario Stoppani, Milano, 31 gennaio 1864.

30 Ibid.

31 Ibid.

32 Ibid.

33 Ibid.

34 Casalena M.P., 2007: 13.

35 Ibid.

36 Biblioteca MSNM, Fondo Cornalia, b. 13, fasc. 6, circolare a stampa, cit.

37 Archivio SISN, b. 1859-1869, Carteggio generale 1859-1865, lettera di Caruel a Cornalia, Firenze, 21 febbraio 1864.

38 Ibid.

39 Archivio SISN, b. 1859-1869, Carteggio generale 1859-1865, lettera di Federico Lancia di Brolo al presidente Cornalia, Palermo, 26 marzo 1864.

40 Ibid.

41 Ibid.

42 Archivio SISN, b. 1859-1869, Carteggio generale 1859-1865, lettera del presidente della Società agraria di Lombardia Alfonso Litta Modignani e del segretario Francesco Pavesi alla SISN, Milano, 2 agosto 1864.

43 Stoppani A., 1863: 406-407.

44 Ibid.: 407.

45 Sella Q., 1864: 56.

46 Ibid.: 57.

47 Ibid.: 58. 
48 Si trattava del decreto reale del 28 luglio 1861, con il quale era stata istituita una Giunta consultiva di esperti - con relatore Giovanni Capellini (1833-1922) - incaricata di stabilire i criteri di compilazione della carta geologica del nuovo Regno d'Italia. Tra i suoi membri figuravano anche i naturalisti milanesi Giulio Curioni (1796-1878), Giovanni Omboni e Antonio Stoppani, soci fondatori della SISN. I lavori della Giunta, animati principalmente da Igino Cocchi (1827-1913) e da Quintino Sella, si erano svolti a Firenze nel settembre 1861, in occasione dell'Esposizione nazionale e del Congresso dell'Accademia dei Georgofili.

49 Sella Q., 1864: 60.

50 Biblioteca MSNM, Fondo Cornalia, b. 2, fasc. 42, De Filippi Filippo, lettera di De Filippi a Cornalia, s.1., [1864] e fasc. 11, Casati Gabrio, lettera di Casati a Cornalia, Biella, 14 settembre 1864.

51 Cornalia E., 1864: 81-83.

$52 \quad$ Ibid.: 87

53 Ibid.: 88.

54 Ibid.: 83.

55 Ibid.: 83-84.

56 Ibid.: 84

57 Ibid.: 87.

58 Intervento di Cornalia. Atti SISN, 4 (1864), seduta 27 novembre 1864: 554.

59 Riunione Biella, 1864.

60 L'articolo 17 del regolamento, infatti, prevedeva che "fra le letture, sarà accordata la preferenza a quelle che hanno per oggetto l'illustrazione del paese in cui si tiene l'adunanza" (Regolamento 1865: 553). La tradizione di descrivere il paese in cui si tenevano i congressi risaliva ai congressi degli scienziati italiani preunitari. Si deve tuttavia Carlo Cattaneo l'idea di descrivere l'intera regione dal punto di vista geologico-naturalistico e sociale, anziché fornire una classica guida storico-artistica della singola città: nacquero così le Notizie naturali e civili su la Lombardia, da lui pubblicate - con la stretta collaborazione dei naturalisti milanesi - presso Bernardoni di Milano in occasione del sesto Congresso degli scienziati italiani del 1844.

61 Riunione Spezia, 1865: 262.

62 Ibid.: 295-296. Per il contributo dei naturalisti alla nascita dell'archeologia preistorica cfr. Tarantini M., 2000.

63 Castelfranco P., 1899: 221-222.

64 In occasione della seconda riunione straordinaria della Società italiana di scienze naturali. Corriere del Golfo, Spezia, 20 settembre 1865, supplemento al n. 14: 57 (copia in Biblioteca MSNM, Fondo Cornalia, b. 13, fasc. 6).

65 Ibid

66 Cfr. Atti SISN, 9 (1866): 235.

67 Ibid.: 481-482.

68 Atti SISN, 9 (1866), seduta 31 marzo 1867: 148-149.

69 Lettera di Cornalia alla Giunta municipale di Vicenza, Milano, 8 aprile 1867. Atti SISN, 10 (1867), seduta 31 marzo 1867: 149-150.

70 Atti SISN, 10 (1867), seduta 24 novembre 1867: 400-401.

71 Atti SISN, 10 (1867): 165-166.

72 Atti SISN, 11 (1868): 426.

73 Ibid.: 436 .

74 Ibid.: 437.

75 Ibid.: $364-365$.

76 Ibid: : $372-376$

77 Non sembra infatti avere a che fare con la rivista Malpighia nata nel 1887 a Messina e successivamente spostata a Genova e poi a Bologna.

78 Atti SISN, 11 (1868): 361.

79 Atti SISN, 12 (1869), seduta 31 gennaio 1869: 144.

80 Ibid.

81 Ibid.: 146.

82 Ibid.: 147.

83 Atti SISN, 12 (1869), seduta 29 aprile 1869: 159.

84 Riunione Catania, 1869: 418-419.

85 Aradas A., 1869: 501. 
86 Atti SISN, 13 (1870), seduta 27 febbraio 1870: 53-54.

87 Ibid.: 54.

88 Ibid.

89 Atti SISN, 14 (1871), seduta 25 giugno 1871: 111.

90 Ibid.: 116-117.

91 Atti SISN, 14 (1871), seduta 6 ottobre 1871: 141.

92 Marinoni C., 1871.

93 Marinoni C., 1872: 266-272.

94 Ibid.: 267.

95 Ibid.: 268.

96 Ibid.: 269.

97 Ibid.: 270.

98 Atti SISN, 15 (1872), seduta 29 dicembre 1872: 502.

99 Atti SISN, 16 (1873), seduta 27 luglio 1873, lettera circolare ai soci del 5 agosto 1873: 140.

100 Stoppani A., 1883: 18.

101 Atti SISN, 17 (1874), sedute 28 giugno e 26 luglio 1874: 212 e 261.

102 Sordelli F., 1874.

103 Riunione Varese, 1878, Adunanza generale d'apertura, 24 settembre 1878: 200.

104 Maggi L., 1878: 275.

105 Riunione Varese, 1878: 212.

106 Ibid.: 253.

107 Pini N., 1885: 23.

108 Cfr. Casalena M.P., 2007: 228.

109 Pini N., 1885: 22.

110 Atti SISN, 26 (1883), seduta 29 aprile 1883: 100.

111 Ibid.

112 Pini N., 1885: 26.

113 Atti SISN, 26 (1883), seduta 29 aprile 1883: 101.

114 Ibid., seduta 1 luglio 1883: 372.

115 Cfr. Canadelli E., 2008.

116 Regolamento 1895: 17, art. 14.

117 Atti SISN, 35 (1895), seduta 1 dicembre 1895: 281.

118 Regolamento 1895: 16, art. 65.

119 Atti SISN, 35 (1895), seduta 7 febbraio 1895: 192.

120 Ibid., seduta 1 dicembre 1895: 282-283.

121 Ibid.: 283.

122 Atti SISN-MSNM, 36 (1896), seduta 12 gennaio 1896: 46.

123 Atti SISN-MSNM, 37 (1897-98), seduta 28 novembre 1897: 119-120.

124 Atti SISN-MSNM, 38 (1899), seduta 27 novembre 1898: 206.

125 Atti SISN-MSNM, 39 (1900), sedute 18 giugno e 17 dicembre 1899.

126 Ibid., seduta 25 novembre 1900: 368.

127 Atti SISN-MSNM, 43 (1904), seduta 21 febbraio 1904: 23.

128 Atti Congresso 1906.

129 Ibid.: 4.

130 Ibid.: 20 .

131 Ibid.: 21.

132 Si vedano le notizie sulla nascita della SIPS nel sito www.sipsinfo.ite Casalena M.P., 2007:231-236.

133 Atti Congresso 1906: 23.

134 Atti SISN-MSNM, 45 (1906), seduta 18 novembre 1906: 24.

\section{Bibliografia}

Aradas A., 1869 - Abbozzo del panorama etneo. Atti della Società italiana di Scienze naturali, 12: 499-534.

Atti del Congresso dei naturalisti italiani promosso dalla Società Italiana di Scienze Naturali, Milano, 15-19 settembre 1906, 1907. Tipografia degli Operai, Milano. 
Atti della Società Geologica residente in Milano. Bernardoni, Milano, 1855-1859.

Atti della Società Italiana di Scienze Naturali. Bernardoni, Milano, 1860-1895.

Atti della Società Italiana di Scienze Naturali e del Museo Civico di Storia Naturale in Milano. Bernardoni, Milano, 1896-1902; Tipografia degli operai, Milano, 1903-1906.

Brianta D. \& Laureti L., 2006 - Cartografia, scienza di governo e territorio nell'Italia liberale. Unicopli, Milano.

Brianta D., 2007 - Europa mineraria. Circolazione delle élites e trasferimento tecnologico (secoli XVIII-XIX). Franco Angeli, Milano.

Canadelli E., 2008 - Alla ricerca del coordinamento. Dal "grande Politecnico" all'“Università politecnica". In: Milano scientifica 1875-1924. La rete del grande Politecnico. Canadelli E. \& Zocchi P. (a cura di). Sironi Editore, Milano, 1: $17-45$.

Canadelli E., 2009 - Tito Vignoli da professore di antropologia a direttore del Museo civico di storia naturale, Milano città delle scienze <www.milanocittadellescienze.it.> maggio 2011

Casalena M. P., 2006 - Opposizione e integrazione. La scienza nazionale nelle capitali e nelle province (XVIII-XIX). Storicamente, 2. <www.storicamente.org.>

Casalena M. P., 2007 - Per lo Stato, per la Nazione. I congressi degli scienziati in Francia e in Italia (1830-1914). Carocci, Roma.

Castelfranco P., 1899 - Archeologia e paletnologia. Atti della Società italiana di Scienze naturali e del Museo civico di Storia naturale in Milano, 38 (1899): 211-231.

Ciardi M., 2010 - Reazioni tricolori. Aspetti della chimica italiana nell'età del Risorgimento. Franco Angeli, Milano.

Cornalia E., 1859a - Programma di studii proposto alla Società dal professore Cornalia. Atti della Società Geologica residente in Milano, 1 (1855-1859), seduta 23 gennaio 1859: 70-78.

Cornalia E., 1859b - Commemorazione del socio Torquato Canetta. Atti della Società Geologica residente in Milano, 1 (1855-1859), seduta 21 agosto 1859: 546-548.

Cornalia E., 1860 - Sulla vita e sulle opere di Abramo Massalongo. Atti della Società italiana di Scienze naturali, 2 (1859-1860), seduta 22 luglio 1860: 200-202.

Cornalia E., 1864 - Sull'origine e sullo sviluppo della Società italiana di scienze naturali. Atti della Società italiana di Scienze naturali, 7 (1864): 81-89.

Corsi P., 2003 - La Carta Geologica d'Italia: agli inizi di un lungo contenzioso. In: Four centuries of the word Geology. Ulisse Aldrovandi 1603 in Bologna. Vai G. B. \& Cavazza W. (a cura di). Minerva Edizioni, Bologna: 255-279.

Govoni P., 2002 - Un pubblico per la scienza. La divulgazione scientifica nell'Italia in formazione. Carocci, Roma.

In occasione della seconda riunione straordinaria della Società Italiana di Scienze Naturali. Corriere del Golfo, Spezia, 20 settembre 1865, supplemento al n. 14: 57.

Livi P., 2008a - Il Museo civico di storia naturale tra collezioni, didattica e ricerca sperimentale. In: Milano scientifica 1875-1924, La rete del grande Politecnico. Canadelli E. \& Zocchi P. (a cura di). Sironi, Milano, 1: 119-138.

Livi P., 2008b - La storia naturale dell'uomo nella Milano dell'Ottocento. Un viaggio attraverso le raccolte del Museo civico di storia naturale. Atti della Società italiana di Scienze naturali e del Museo civico di Storia naturale in Milano, 149 (2): 273-292. 
Maggi L., 1878 - Intorno alle condizioni naturali del territorio varesino. Discorso letto nell'apertura solenne della VII Riunione straordinaria della Società di scienze naturali in Varese il dì 24 settembre 1878. Atti della Società italiana di Scienze naturali, 21 (1878): 273-300.

Marinoni C., 1871 - Sul quinto Congresso internazionale di antropologia e di archeologia preistorica, tenuto a Bologna nell'ottobre 1871. Atti della Società italiana di Scienze Naturali, 14 (1871): 228-240.

Marinoni C., 1872 - Sullo stato morale e materiale della Società italiana di scienze naturali nell'anno 1872. Atti della Società italiana di Scienze Naturali, 15 (1872): 266-272.

Omboni G., 1860 - Il Congresso dei naturalisti svizzeri in Lugano nel settembre 1860. Atti della Società italiana di Scienze Naturali, 2 (1859-1860), seduta 25 novembre 1860: 312-333.

Parisi B., Franchino A. \& Berti A., 2000 - La Società Italiana di Scienze Naturali 1855-2000. Percorsi storici e documenti. Natura della Società italiana di Scienze naturali e del Museo civico di Storia naturale di Milano, 90 (1).

Piccioni L., 1999 - Il volto amato della patria. Il primo movimento per la protezione della natura in Italia 1880-1934. Dipartimento di botanica ed ecologia, Università degli studi di Camerino, Camerino.

Pini N., 1885 - Relazione sullo stato morale e materiale della Società dalla sua fondazione al 30 giugno 1884. Atti della Società italiana di Scienze Naturali, 28 (1885): 16-31.

Quazza G. \& Quazza M. (a cura di), 1980 - Epistolario di Quintino Sella, vol. 1: (1842-1865). Istituto per la storia del Risorgimento italiano, Roma.

Sella Q., 1864 - Discorso d'apertura del Congresso dei naturalisti pronunziato dal presidente straordinario comm. Quintino Sella nella tornata del 3 settembre 1864 in Biella. Atti della Società italiana di Scienze naturali, 7 (1864): 55-80.

Società Italiana di Scienze Naturali - Regolamento generale approvato da S.E. il Ministro dell'Interno il 21 luglio 1858. Atti della Società Geologica residente in Milano, 1 (1855-1859): 23-30.

Società Italiana di Scienze Naturali - Regolamento per le riunioni straordinarie approvato nella seduta ordinaria del 3 aprile 1864 e modificato nella seduta del 26 marzo 1865. Atti della Società italiana di Scienze naturali, 8 (1865): 551556.

Società Italiana di Scienze Naturali - Regolamento della Società italiana di scienze naturali colle modificazioni approvate nelle adunanze dei giorni 28 marzo e 2 maggio 1895. Atti della Società italiana di Scienze naturali, 35 (1895): IVXVII.

Società Italiana di Scienze Naturali - Società italiana di scienze naturali in Milano. Riunione straordinaria in Biella nei giorni 3, 4, 5 e 6 settembre 1864. Giornale dell'ingegnere-architetto ed agronomo, 12 (ott. 1864): 633-634.

Società Italiana di Scienze Naturali - Seconda riunione straordinaria della Società italiana di scienze naturali tenuta alla Spezia nei giorni 18, 19, 20 e 21 settembre 1865. Atti della Società italiana di Scienze naturali, 8 (1865).

Società Italiana di Scienze Naturali - Quarta riunione straordinaria della Società italiana di scienze naturali tenuta in Catania nei giorni 23, 24, 25 e 26 agosto 1869. Atti della Società italiana di Scienze naturali, 12 (1869): 409-498.

Società Italiana di Scienze Naturali - Settima riunione straordinaria di Varese. Atti della Società italiana di Scienze naturali, 11 (1878). 
Sordelli F., 1874 - Relazione sulla adunanza dei naturalisti italiani tenuta in Arco (Trentino) nei giorni 21-24 settembre, fatta alla Società italiana di scienze naturali nella seduta del 29 novembre 1874. Atti della Società italiana di Scienze naturali, 17 (1874): 455-462.

Stoppani A., 1863 - Il Congresso dei naturalisti svizzeri a Samaden. Atti della Società italiana di Scienze naturali, 5 (1863), seduta 6 dicembre 1863: 406415.

Stoppani A., 1883 - Inaugurazione nel Museo civico di Milano del ricordo monumentale a Emilio Cornalia, 8 giugno 1883. Bernardoni, Milano.

Tarantini M., 2000 - Tradizioni e tensioni disciplinari nell'archeologia preistorica italiana tra Otto e Novecento. Origini, 23: 7-43.

Vaccari E., 1999 - Austro-Italian Scientific Relationship in the field of the Earth Sciences. The Geologische Reichsanstalt and the geologists of the LombardoVenetian Kingdom (1850-1866). Abhandlungen der Geologischen Bundesanstalt, 56 (1): 95-114.

Zocchi P., 2010a - La fondazione della Società Geologica residente in Milano (poi Società Italiana di Scienze Naturali) e i suoi rapporti internazionali in un carteggio italo-austriaco. Milano città delle scienze. $<$ www.milanocittadellescienze. it.>

Zocchi P., 2010b - Il Museo Civico di Storia Naturale di Milano, 1'Istituto geologico di Vienna e le premesse per la costituzione della Società geologica residente in Milano. Milano città delle scienze. <www.milanocittadellescienze.it.>

Zocchi P., 2010c - Il Museo Civico di Storia Naturale di Milano e l'associazionismo scientifico tra Otto e Novecento. Milano città delle scienze. <www.milanocittadellescienze.it.>

\section{Fonti archivistiche}

Museo Civico di Storia Naturale di Milano, Archivio storico (Archivio MSNM). Archivio amministrativo, busta 11.

Museo Civico di Storia Naturale di Milano, Biblioteca (Biblioteca MSNM). Fondo Cornalia, buste 2 e 13 .

Società Italiana di Scienze Naturali. Archivio storico (Archivio SISN), buste 18551859 e 1859-1869. L'archivio non è riordinato ed è attualmente chiuso al pubblico. La documentazione è stata gentilmente messa a disposizione dalla SISN per questa ricerca.

Ricevuto: 29 marzo 2011

Approvato: 15 aprile 2011 